\title{
Trend of Unintended Pregnancy, Induced Abortion and Associated Factors among Adolescents in Ethiopia: Evidence from the 2000, 2005, 2011 and 2016 EDHS Data
}

\author{
Tiruwork Amare \\ Addis Ababa City Administration Health Bureau \\ Fasil Tessema \\ Jimma University \\ Tamrat Shaweno ( $\sim$ babiynos@gmail.com ) \\ Africa Centres for Disease Control and Prevention
}

\section{Research Article}

Keywords: Unintended pregnancy, induced abortion, Adolescent, Ethiopia

Posted Date: February 2nd, 2022

DOI: https://doi.org/10.21203/rs.3.rs-1204114/v1

License: (c) (i) This work is licensed under a Creative Commons Attribution 4.0 International License. Read Full License 


\section{Abstract \\ Background}

Adolescents are highly at risk of unintended pregnancy due to physiological, sexual, social and psychological growth. The pregnancy may end with early childbirth, induced abortion and its complications. Trends of unintended pregnancy and induced abortion are higher in developing countries and more than $90 \%$ of adolescent births occur in low income countries.

\section{Objectives}

This study determined the trend of unintended pregnancy, induced abortion and associated factors among adolescents in Ethiopia from 2000 to 2016.

\section{Methods}

Trends in the prevalence of unintended pregnancy and abortion among adolescents were investigated based on a series of the Ethiopia Demographic and Health Surveys (EDHS) data for the years $2000(n=741), 2005(n=708), 2011$ ( $n=616)$, and $2016(n=479)$. The combined dataset was used to increase the statistical power of the study in order to detect any association between the study factors and the outcomes, and to examine trends in unintended pregnancy and abortion over the study period (2000-2016). The percentage changes with corresponding $95 \% \mathrm{Cl}$ of the outcome variables were calculated by independent factors to examine the changes over the study period using chi-squared test for trend. Multilevel logistic regression analysis was done to identify factors associated with unintended pregnancy and abortion among adolescents.

\section{Results}

The prevalence of unintended pregnancy among Ethiopian adolescents aged 15-19 years decreased significantly from $41.4 \%$ (95\% Cl: $35.7,47.2 \%)$ in 2000 to $25.1 \%$ (95\% Cl: 18.9, 31.4\%) in 2016 (p-value=0.0001). Factors associated with a lower likelihood of unintended pregnancy among adolescents included being aged above 17 years old ( $\mathrm{AOR}=0.30 ; 95 \% \mathrm{Cl}$ : 0.17-0.51) and married ( $A O R=0.14 ; 95 \% \mathrm{Cl}: 0.07-0.28)$. While, parity ( $\mathrm{AOR}=1.68 ; 95 \% \mathrm{Cl}: 1.02-2.75)$, resided in large central $(A O R=3.32 ; 95 \% \mathrm{Cl}: 1.86-5.63)$, and metropolis (AOR=3.12; 95\% Cl:1.20-8.12) regions were significantly associated with higher odds of untended pregnancy. The prevalence of abortion also decreased significantly from 8.3\% (95\% Cl: 5.2, 11.4\%) in 2000 to $4.1 \%$ (95\% Cl:1.3, 6.9\%) in 2016 (p-value=0.04). Middle wealth index (AOR=0.14; 95\% Cl: 0.03-0.64), rich wealth $(A O R=0.09 ; 95 \% \mathrm{Cl}: 0.01-0.74)$, female head household $(\mathrm{AOR}=0.11 ; 95 \% \mathrm{Cl}: 0.01-0.91)$ and rural resident $(\mathrm{AOR}=0.08 ; 95 \% \mathrm{Cl}$ : $0.01-0.45)$ were factors significantly associated with lower likelihood of abortion.

\section{Conclusion}

Although, the prevalence of unintended pregnancy and abortion among adolescents decreased significantly from 2000 to 2016, still both are public health problems in Ethiopia. Factors associated with higher unintended pregnancy were being below 18 years old, unmarried, higher parity, large central and metropolis regions. Belonging to poorer wealth index, urban resident and male headed households were factors associated with higher abortion rate. Hence, it is important to provide continuous sex education, contraception education and health information about the consequences of unintended pregnancy and abortion for adolescent girls in Ethiopia.

\section{Introduction}


Unintended pregnancies are pregnancies that are mistimed, unplanned or unwanted at the time of conception [1]. Unintended pregnancies may also result from rape, incest or various other forms of forced or unwanted sex [2, 3]. Unintended pregnancy is a global public health problem and its sequels are major causes for maternal morbidity and mortality with its effect to maternal illness as well [4-6]. Globally, unintended pregnancy has a critical public, clinical and social health concern because it commonly results in induced abortion whether it's safe or unsafe leads to complication and maternal death. It has been reported that of the estimated 210 million pregnancies that occur each year worldwide, approximately $38 \%$ are unplanned and $22 \%$ are terminated [7].

Induced abortion is the termination of a fetus that brought about intentionally using different options safe or unsafe based on quality of care. It doesn't include spontaneous and other post abortion cares that comes accidentally or due to other causes when the pregnancy is intended [7, 8]. Globally, the estimated rate in the period 2010-2014 was 35 abortions per 1000 women (aged 15-44 years) and an estimated 21.6 million unsafe abortions took place worldwide in 2008 almost all in developing countries [5]. Abortion rates have declined significantly since 1990 in the developed world but not in the developing world [7, 9]. Induced abortion and unintended pregnancy rates are increasing in developing regions than developed regions [9]. Ensuring access to sexual and reproductive healthcare could help millions of women avoid unintended pregnancies and ensure access to safe abortion [7].

Adolescent is a transitional phase of growth and development between childhood and adulthood [10] between the age of 10 and 19 years [11]. Adolescents are highly at risk of unintended pregnancy due to physiological, sexual, social and psychological growth [12]. The pregnancy may end with early childbirth, induced abortion and its complications [13]. In Ethiopia, an estimated 620,300 induced abortions were performed in 2014. The annual abortion rate among women aged 15-49 was 28 per 1,000 that showed an increase from 22 per 1,000 in 2008, and was highest in urban than rural areas [14]. Each year, about 79 million unintended pregnancies, excluding miscarriage, occur worldwide. More than half of these unintended pregnancies end in abortion [15]. Data on abortion rates are inexact but can be used to explore trends. Unintended pregnancy rates have declined worldwide but vary widely across regions. In many areas of the world where rates of unintended pregnancy are high [16,17], and unsafe abortions have also been shown to be high [18]. Of all pregnancies worldwide in $2008,41 \%$ were reported as unintended or unplanned, and approximately $50 \%$ of these ended in abortion. Of the estimated 21.6 million unsafe abortions occurring worldwide in 2008 (around one in 10 pregnancies), approximately 21.2 million occurred in developing countries, often due to restrictive abortion laws and leading to an estimated 47,000 maternal deaths and untold numbers of women who will suffer long-term health consequences [16].

In Africa, the importance of knowing the trends of unintended pregnancy and induced abortion is very vital. Because different literatures show us, in developing countries there is high number of unintended pregnancy and abortion, whether safe or unsafe. In Malawi, 53\% pregnancies are unintended and that of 30\% ends with abortion which is 2015 [19]. Similarly, higher rate of abortion was reported in Nigeria [20] and in Congo [21].

Ethiopia's parliament amended the criminal code of the federal democratic republic of Ethiopia the (Proclamation No. 414/2004) to expand the circumstances in which abortion is legal in 2005 and the country has expanded abortion care service and post abortion family planning [22]. One in three birth is unintended (32\%) and about two third of these were mistimed [23]. Unintended pregnancy has a multiple determinates and leads to an induced abortion and affects maternal health, and may end with morbidity and mortality[4, 5].

Results from EDHS 2016 reported $22 \%$ unmet need for family planning of which $9 \%$ was for limiting and spacing. The contraceptive prevalence rate trend has shown a significant change from 6\% in 2000 to 35\% in 2016. But there are factors associated with it and needs further studies [24]. Information on trends of unintended pregnancy and induced abortion has a great role in developing strategies and prevention modalities for complications due to unintended pregnancy and unsafe abortion where most maternal injuries and deaths are related to unsafe abortion specially in developing regions including 
Ethiopia. Thus, this study aimed to determine the trend of unintended pregnancy, induced abortion and associated factors among adolescents in Ethiopia from 2000 to 2016.

\section{Methods}

\section{Data source}

The study utilized data from cross sectionally conducted four consecutive Ethiopian Demographic and Health Surveys of 2000, 2005, 2011 and 2016 EDHS women's data (IR data is the EDHS women's data file code) set in Ethiopia. According to the Ethiopian census report; by the year 2018 the estimated population of Ethiopia were 106.8 million of this $24.2 \%$ were adolescent (from 9 to 18 years) and $11.7 \%$ of them were 15 to 19 year old. Adolescent girls were $5.8 \%$ from a total population. The fertility rate was 56.61 births per 1000 women aged 15-19 years old [25]. Until now, four surveys were conducted in Ethiopia where reports are available in CSA and online [26]. The first survey was conducted in 2000/1 followed by the 2005/6 EDHS which is part of the worldwide measure DHS project funded by USAID and was conducted under the auspices of the ministry of health and implemented by the then Population and Housing Census Commission Office (PHCCO). The EDHS surveys of 2011 and 2016 are also the same surveys like other years. The objective of EDHS is for planning, policy formulation, monitoring, and evaluation of population and health program in the country [24].

\section{Sample size determinations and sampling procedure}

Our analysis was based on women's data (IR data) set for EDHS 2000, 2005, 2011 and 2016. The EDHS used a two-stage stratified cluster sampling technique to select the study participants. In stage one, after each administrative region was stratified into urban and rural strata, Enumeration Areas (EAs) were selected using a probability proportional to EA size. In stage two, a household listing operation was carried out in all of the selected EAs and a fixed number of households from each EA were selected. All women aged 15-49 years who were permanent residents or who spend the night in the selected households the night before the survey were included in the surveys [24]. In this study, a total weighted sample of 2544 adolescents (15-19 years old) who gave birth in the 5 years preceding the surveys or pregnant during the surveys were included. Trends in the prevalence of unintended pregnancy and abortion were investigated based on a series of the Ethiopia Demographic and Health Surveys (EDHS) data for the years 2000 (n=741), $2005(n=708), 2011(n=616)$, and 2016 $(n=479)$.

\section{Study variables}

The dependent variables considered were unintended pregnancy and abortion. Unintended pregnancy is defined as a pregnancy which is a sum of mistimed pregnancy (pregnancy wanted at a later time) and unwanted pregnancy (pregnancy which is not wanted at all [3]. Abortion was taken from the EDHS question 'have you ever had a pregnancy termination?' with the response 'yes' if the woman ever had an abortion and otherwise 'no' as a binary outcome. The independent variables included in this study were socio-demographic variables like maternal age, educational status, occupation status, marital status, religion, wealth status, residence, region and household size. Reproductive health variables such as parity, birth order, age at first sex, antenatal care visit, place of delivery, knowledge of ovulation cycle, distance from the health facility, involving in health care decision, media exposure, knowledge and practice of family planning. Variables were recoded to make suitable for the study analysis such as region; Afar, Somali, Benishangul, and Gambela as "small peripheral regions", Tigray, Amhara, Oromia, and Sothern Nations Nationalities and Peoples Region (SNNPR) as "large central regions" and Harari, Dire Dawa, and Addis Ababa as "metropolitans", based on their geopolitical features [27].

\section{Data extraction instrument}

The EDHS tool has three components: the household questionnaire, the woman's questionnaire and the man's questionnaire. These questionnaires were adapted from model survey instruments developed for the MEASURE DHS project to reflect the population and health issues relevant to Ethiopia [24]. The data for this study were taken from woman's 
questionnaire. The variables reviewed to be important for this specific research were collected from the SPSS filled data received from http:// www.measuredhs.com and these data were transferred into a Stata Software version 14.

\section{Data processing and analysis}

Preliminary analyses involved the description of the study participants by calculating frequencies and percentages of the study variables (socioeconomic, demographic, reproductive health services). This was followed by the estimation of the prevalence of the outcome variables (unintended pregnancy and abortion) and by socioeconomic and reproductive health variables in both years' specific data $(2000,2005,2011$ and 2016) and in the combined dataset [24]. Then, percentage point change with corresponding $95 \% \mathrm{Cl}$ of the outcome variables was calculated by each of the study factors to examine the changes over the research period from 2000 to 2016. We used the combined dataset to increase the statistical power of the study in order to detect any association between the study factors and the outcomes, as well as to examine trends in unintended pregnancy and abortion over the study period (2000-2016). We estimated P for trends in each category of the study variables to assess whether the prevalence decreased or increased over the study period using chi-squared test for trend.

A multi-level logistic regression analysis was applied to account for the hierarchal nature of the DHS data and bivariable multilevel logistic regression analysis was carried to determine the crude odds ratios at $95 \%$ confidence interval and those variables with $\mathrm{p}$-value $<0.25$ were considered for multivariable analysis. In the multivariable multilevel logistic analysis, those variables with $\mathrm{p}$-value $<0.05$ were considered as significantly associated with outcome variable. After selecting variables for multivariable analysis, four models containing variables of interest were fitted. These models were model 1 is the null model in which no factor variables were included, model 2 which examined the effects of individual-level factors, model 3 examined the effect of community level factors and model 4 which examined the effects of both individual and community level variables. The Intra-Class Correlation (ICC) was estimated to assess the cluster effect and the model fitness was compared using information criteria (IC). All statistical analyses were conducted using Stata version 14.0 with 'svy' command to adjust for sampling weights, clustering effects and stratification, and the 'melogit' command was used for the regression analysis. Finally, the results were presented with tables and graph.

\section{Data quality and assurance}

The data quality depends on EDHS data quality and the formats that are going to be use like data extraction formats. The quality of the data was maintained by checking its completeness, cleaning the missing values by running frequencies and some of the variables were re-coded.

\section{Results}

\section{Socio demographic and economic characteristics}

In this study, a total weighted sample of 2544 women age 15-19 years who gave birth in the 5 years preceding the surveys or pregnant during the surveys were included. About three-fourth of study participants were 18 and 19 years of age (75.5\%) and the overall mean age was 18 years. Majority of respondents, $60.8 \%$ had no formal education and $45.3 \%$ of them were orthodox Christians. Around $60.7 \%$ of participants were unemployed and $57.6 \%$ of women were from poor household wealth status. Most, $89.8 \%$ of women were married while only $43.5 \%$ of them had media exposure. Regarding household size, $75.7 \%$ of the respondents were from a household size of two to five. Majority of the women were rural residents $(91.1 \%)$ and from large central regions (92.2\%). Distance to health facility was a big problem for $71.2 \%$ of respondents and $55.6 \%$ of them not involved in decision for health care (Table 1). 
Table 1

Socio demographic and economic characteristics of adolescents in Ethiopia, 2000-2016.

\begin{tabular}{|c|c|c|c|}
\hline \multicolumn{2}{|c|}{ Socio demographic and economic variables } & \multirow{2}{*}{$\begin{array}{l}\text { Frequency } \\
623\end{array}$} & \multirow{2}{*}{$\begin{array}{l}\text { Percent } \\
24.5\end{array}$} \\
\hline Age & $15-17$ & & \\
\hline & $18-19$ & 1921 & 75.5 \\
\hline \multirow[t]{2}{*}{ Residence } & Urban & 228 & 8.9 \\
\hline & Rural & 2316 & 91.1 \\
\hline \multirow[t]{3}{*}{ Educational status } & No education & 1547 & 60.8 \\
\hline & Primary & 871 & 34.2 \\
\hline & Secondary and higher & 125 & 4.9 \\
\hline \multirow[t]{2}{*}{ Marital status } & Not married & 260 & 10.2 \\
\hline & Married & 2284 & 89.8 \\
\hline \multirow[t]{4}{*}{ Religion } & Orthodox & 1148 & 45.3 \\
\hline & Protestant & 389 & 15.3 \\
\hline & Muslim & 946 & 37.3 \\
\hline & Others & 54 & 2.1 \\
\hline \multirow[t]{2}{*}{ Employment status } & Not working & 1453 & 60.7 \\
\hline & Working & 940 & 39.3 \\
\hline \multirow[t]{3}{*}{ Family size } & $2-3$ & 1079 & 42.4 \\
\hline & $4-5$ & 846 & 33.3 \\
\hline & $>=6$ & 619 & 24.3 \\
\hline \multirow[t]{3}{*}{ Household wealth } & Poor & 1395 & 57.6 \\
\hline & Middle & 694 & 28.6 \\
\hline & Rich & 335 & 13.8 \\
\hline \multirow[t]{3}{*}{ Regions category } & Large central & 2344 & 92.2 \\
\hline & Small peripheral & 150 & 5.9 \\
\hline & Metropolis & 50 & 1.9 \\
\hline \multirow[t]{7}{*}{ Regions } & Tigray & 177 & 6.9 \\
\hline & Afar & 31 & 1.2 \\
\hline & Amhara & 678 & 26.7 \\
\hline & Oromia & 1176 & 46.2 \\
\hline & Somali & 74 & 2.9 \\
\hline & Benishangul-Gumuz & 34 & 1.4 \\
\hline & SNNPR & 313 & 12.3 \\
\hline
\end{tabular}




\begin{tabular}{|c|c|c|c|}
\hline \multicolumn{2}{|c|}{ Socio demographic and economic variables } & \multirow{2}{*}{$\begin{array}{l}\text { Frequency } \\
11\end{array}$} & \multirow{2}{*}{$\begin{array}{l}\text { Percent } \\
0.4\end{array}$} \\
\hline & Gambela & & \\
\hline & Harari & 8 & 0.3 \\
\hline & Addis Ababa & 33 & 1.3 \\
\hline & Dire Dawa & 9 & 0.4 \\
\hline \multirow[t]{2}{*}{ Media exposure } & No & 1438 & 56.5 \\
\hline & Yes & 1106 & 43.5 \\
\hline \multirow[t]{2}{*}{ Distance to health facility } & Big problem & 1283 & 71.2 \\
\hline & Not a big problem & 521 & 28.8 \\
\hline \multirow[t]{2}{*}{ Decision for health care } & Not involved & 1413 & 55.6 \\
\hline & Involved & 1131 & 44.4 \\
\hline
\end{tabular}

\section{Reproductive history of the study participants}

Among 2544 total study respondents, about two-third (67.6\%) of women had one live child, $28.2 \%$ had two or more children while $4.2 \%$ had no live birth child. About two-third (66.0\%) of respondents were fifteen and younger years old during their first sex. About $38.5 \%$ of respondents had at least one Antennal care (ANC) visit history, and only $13.0 \%$ of study participants delivered their child in a health facility. Most, $89.8 \%$ of woman knew at least one family planning method while $13.4 \%$ of them correctly knew that a women is most likely to conceive half way between two periods and only $23.1 \%$ of them had ever used contraceptive. Among 2544 total study respondents, 1677 (66.0\%) had wanted then (planned) pregnancies, $657(25.9 \%)$ of them had wanted later (miss-timed) pregnancies while $206(8.1 \%)$ had wanted not at all pregnancies. While, $132(5.2 \%$ ) of respondents had abortion (Table 2). 
Table 2

Reproductive history of adolescents in Ethiopia, 2000-2016.

\begin{tabular}{|c|c|c|c|}
\hline Variables & & Frequency & Percent \\
\hline \multirow[t]{4}{*}{ Parity } & 0 & 107 & 4.2 \\
\hline & 1 & 1719 & 67.6 \\
\hline & 2 & 609 & 23.9 \\
\hline & $3-4$ & 109 & 4.3 \\
\hline \multirow[t]{2}{*}{ ANC visit history } & No & 1312 & 61.5 \\
\hline & Yes & 822 & 38.5 \\
\hline \multirow[t]{2}{*}{ Place of delivery } & Home & 1865 & 87.0 \\
\hline & Health facility & 278 & 13.0 \\
\hline \multirow[t]{2}{*}{ Mode of delivery } & Non- Caesarean & 2116 & 98.8 \\
\hline & Caesarean Section & 26 & 1.2 \\
\hline \multirow[t]{3}{*}{ Birth order } & $1 \mathrm{st}$ & 2027 & 79.7 \\
\hline & 2nd & 447 & 17.6 \\
\hline & 3rd or 4th & 70 & 2.7 \\
\hline \multirow[t]{3}{*}{ Pregnancy plan status } & wanted then & 1677 & 66.0 \\
\hline & Wanted later & 657 & 25.9 \\
\hline & Not wanted at all & 206 & 8.1 \\
\hline \multirow[t]{2}{*}{ Knows any family planning } & No & 260 & 10.2 \\
\hline & Yes & 2283 & 89.8 \\
\hline \multirow[t]{2}{*}{ Ever use contraceptive } & No & 1957 & 76.9 \\
\hline & Yes & 587 & 23.1 \\
\hline \multirow[t]{2}{*}{ Knows ovulation period } & Yes & 342 & 13.4 \\
\hline & No & 2201 & 86.6 \\
\hline \multirow[t]{2}{*}{ Age at first sex(years) } & $<=15$ & 891 & 66.0 \\
\hline & $>15$ & 460 & 34.0 \\
\hline \multirow[t]{2}{*}{ Abortion } & No & 2412 & 94.8 \\
\hline & Yes & 132 & 5.2 \\
\hline
\end{tabular}

\section{Prevalence of unintended pregnancy among adolescents in Ethiopia}

Over the study period (2000-2016), the prevalence of unintended pregnancy was $34.0 \%(95 \% \mathrm{Cl}: 31.0-37.1 \%)$. It was $37.6 \%$ in urban areas and $30.6 \%$ in rural areas. The highest prevalence of unintended pregnancy was observed among unmarried adolescents (57.2\%), followed by among women who resided in Addis Ababa (47.1\%), women aged 15-17 years (44.3\%), among women who held secondary and above education (43.4\%) and women from Amhara region (40.9\%). The lowest unintended pregnancy prevalence was found among women from the Somali region (4.2\%), followed by women from Afar region (20.1\%) and Tigray region (22.5\%) (Table 3). Prevalence of unintended pregnancy was also varied by reproductive 
history variables. Relatively higher prevalence of unintended pregnancy was found among women from female headed households (38.5\%), women who had no media exposure (37.9\%), women who had 2-4 live children (37.7\%) and women who had no ANC visit history (37.1\%). While relatively lower prevalence of unintended pregnancy was observed among women who had abortion (15.5\%), who had ANC visit history (21.9\%) and health facility delivery history (26.9\%) (Table 4)

\section{Trends in unintended pregnancy among adolescents in Ethiopia}

The prevalence of unintended pregnancy among Ethiopian adolescents aged 15-19 years decreased significantly from $41.4 \%$ (95\% Cl: 35.7, 47.2\%) in 2000 to $25.1 \%$ (95\% Cl: 18.9, 31.4\%) in 2016 (Fig. 1].

Between the study period 2000 to 2016, the largest significant reduction in unintended pregnancy was observed among women with primary education ( $\mathrm{Diff}=-32.3 ; 95 \% \mathrm{Cl}:-48.4,-16.4$ ), followed by those reside in metropolis regions (Diff $=-29.0 ; 95 \% \mathrm{Cl}:-49.8,-8.3$ ), those who had ever used contraceptive (Diff $=-27.1 ; 95 \% \mathrm{Cl}:-43.4,-10.7)$ and whose age at first sex older than 15 years (Diff $=-26.3 ; 95 \% \mathrm{Cl}:-44.3,-8.4)$.

The prevalence of unintended pregnancy was also significantly reduced from 2000 to 2016 among women lived in Harari (Diff=-25.8; 95\% Cl: -46.7, -4.9), Tigray (Diff=-19.9; 95\% Cl: -36.5, -3.3) and Oromia (Diff=-13.6; 95\% Cl: -27.1, -0.2) regions. It was also significantly reduced among rural residents (Diff $=-17.3 ; 95 \% \mathrm{Cl}$ : $26.3,-8.3$ ), married women (Diff $=-19.0 ; 95 \% \mathrm{Cl}:-27.6,-10.4)$, unemployed women (Diff=-23.5; 95\% Cl: -35.4, -11.5), women from poor household wealth (Diff $=-20.0 ; 95 \% \mathrm{Cl}:-31.0,-9.0)$, women from male headed households (Diff $=-20.9 ; 95 \% \mathrm{Cl}$ : $29.8,-12.1$ ), women who had media exposure (Diff=-19.1; 95\% Cl: $-34.3,-3.9$ ), home delivery (Diff=-18.0; 95\% Cl: $-29.7,-6.3$ ), women who had no abortion (Diff=-18.4; 95\% Cl: $-27.1,-9.7$ ) and those who had ANC history (Diff=-23.9; 95\% Cl: -36.8, -11.0). Similarly, it was significantly reduced among women from 2 to 3 family size (Diff=-15.4; 95\% $\mathrm{Cl}:-28.7,-2.2$ ), from 4-5 family size (Diff=-18.5; 95\% Cl: $-34.9,-2.1)$, women who had one live child (Diff $=-14.9 ; 95 \% \mathrm{Cl}:-24.8,-4.9)$ and women who had 2-4 live children (Diff=-22.9; 95\% Cl: -41.2, -4.7) (Table 3 and Table 4). 
Table 3

Prevalence trend of unintended pregnancy by socio demographic and economic variables among adolescents in Ethiopia, 2000-2016

\begin{tabular}{|c|c|c|c|c|c|c|c|}
\hline Variables & $\begin{array}{l}2000 \\
\%(95 \% \mathrm{Cl})\end{array}$ & $\begin{array}{l}2005 \\
\%(95 \% \mathrm{Cl})\end{array}$ & $\begin{array}{l}2011 \\
\%(95 \% \mathrm{Cl})\end{array}$ & $\begin{array}{l}2016 \\
\%(95 \% \mathrm{Cl})\end{array}$ & $\begin{array}{l}2000- \\
2016 \\
n(\%)\end{array}$ & $\begin{array}{l}2016-2000 \\
\text { aDiff }(95 \% \mathrm{Cl})\end{array}$ & $\begin{array}{l}\text { Trend } \\
\text { p- } \\
\text { value }\end{array}$ \\
\hline \multicolumn{8}{|l|}{ Age(years) } \\
\hline $15-17$ & $\begin{array}{l}55.0(44.4- \\
65.6)\end{array}$ & $\begin{array}{l}45.7(33.8- \\
57.6)\end{array}$ & $\begin{array}{l}37.3(24.8- \\
49-8)\end{array}$ & $\begin{array}{l}34.1(21.8- \\
46.4)\end{array}$ & $276(44.3)$ & $-20.9(-37.2,-4.6)$ & 0.012 \\
\hline $18-19$ & $\begin{array}{l}36.9(30.5- \\
43.3)\end{array}$ & $\begin{array}{l}129.9(23.5- \\
36.4)\end{array}$ & $\begin{array}{l}30.7(24.0- \\
37.3)\end{array}$ & $\begin{array}{l}21.6(14.2- \\
28.9)\end{array}$ & $588(30.6)$ & $-15.3(-25.1,-5.6)$ & 0.002 \\
\hline \multicolumn{8}{|l|}{ Residence } \\
\hline Urban & $\begin{array}{l}43.6(27.8- \\
59.4)\end{array}$ & $\begin{array}{l}40.8(23.5- \\
58.1)\end{array}$ & $\begin{array}{l}23.9(9.1- \\
38.8)\end{array}$ & $\begin{array}{l}38.7(15.9- \\
61.5)\end{array}$ & $86(37.6)$ & $-4.9(-32.6,22.8)$ & 0.728 \\
\hline Rural & $\begin{array}{l}41.1(34.9- \\
47.4)\end{array}$ & $\begin{array}{l}33.4(27.1- \\
39.7)\end{array}$ & $\begin{array}{l}32.7(26.0- \\
39.8)\end{array}$ & $\begin{array}{l}23.8(17.4- \\
30.3)\end{array}$ & $778(33.6)$ & $-17.3(-26.3,-8.3)$ & 0.000 \\
\hline \multicolumn{8}{|l|}{ Education } \\
\hline No education & $\begin{array}{l}36.6(30.2- \\
43.1)\end{array}$ & $\begin{array}{l}31.9(25.1- \\
38.8)\end{array}$ & $\begin{array}{l}25.4(17.3- \\
33.5)\end{array}$ & $\begin{array}{l}16.6(5.6- \\
27.6)\end{array}$ & $479(31.0)$ & $-20.0(-32.8,-7.2)$ & 0.002 \\
\hline Primary & $\begin{array}{l}60.0(46.4- \\
73.8)\end{array}$ & $\begin{array}{l}40.3(28.6- \\
51.9)\end{array}$ & $\begin{array}{l}39.1(30.5- \\
47.6)\end{array}$ & $\begin{array}{l}27.7(19.4- \\
36.0)\end{array}$ & $330(37.9)$ & $\begin{array}{l}-32.3( \\
-48.4,-16.4)\end{array}$ & 0.000 \\
\hline $\begin{array}{l}\text { Secondary \& } \\
\text { higher }\end{array}$ & $\begin{array}{l}58.2(37.8- \\
78.5)\end{array}$ & $\begin{array}{l}27.8(0.9- \\
54.7)\end{array}$ & $\begin{array}{l}25.9(8.9- \\
43.0)\end{array}$ & $\begin{array}{l}40.0(18.1- \\
62.0)\end{array}$ & $54(43.4)$ & $-18.2(-48.1,11.8)$ & 0.235 \\
\hline \multicolumn{8}{|l|}{ Marital status } \\
\hline Not married & $\begin{array}{l}55.9(40.8- \\
76.1)\end{array}$ & $\begin{array}{l}66.9(53.3- \\
80.6)\end{array}$ & $\begin{array}{l}61.7(39.7- \\
83.7)\end{array}$ & $\begin{array}{l}47.0(24.6- \\
69.4)\end{array}$ & $148(57.2)$ & $-8.9(-35.9,18.1)$ & 0.518 \\
\hline Married & $\begin{array}{l}40.0(33.9- \\
46.0)\end{array}$ & $\begin{array}{l}30.9(24.8- \\
37.0)\end{array}$ & $\begin{array}{l}28,9(22.1- \\
35.6)\end{array}$ & $\begin{array}{l}21.0(15.0- \\
27.1)\end{array}$ & 715(31.5) & $-19.0(-27.6,-10.4)$ & 0.000 \\
\hline \multicolumn{8}{|l|}{ Employment } \\
\hline Not employed & $\begin{array}{l}43.8(34.4- \\
53.2)\end{array}$ & $\begin{array}{l}32.0(25.3- \\
38.7)\end{array}$ & $\begin{array}{l}29.7(21.8- \\
37.6)\end{array}$ & $\begin{array}{l}20.3(13.0- \\
27.6)\end{array}$ & $454(31.3)$ & $-23.5(-35.4,-11.5)$ & 0.000 \\
\hline Employed & $\begin{array}{l}39.7(32.0- \\
47.5)\end{array}$ & $\begin{array}{l}41.3(30.5- \\
52.1)\end{array}$ & $\begin{array}{l}34.8(26.4- \\
43.2)\end{array}$ & $\begin{array}{l}32.3(20.7- \\
43.8)\end{array}$ & $348(37.0)$ & $-7.4(-21.4,6.4)$ & 0.292 \\
\hline \multicolumn{8}{|l|}{$\begin{array}{l}\text { Household } \\
\text { wealth }\end{array}$} \\
\hline Poor & $\begin{array}{l}41.1(34.5- \\
47.6)\end{array}$ & $\begin{array}{l}31.6(24.5- \\
38.7)\end{array}$ & $\begin{array}{l}26.4(17.5- \\
35.2)\end{array}$ & $\begin{array}{l}21.1(12.3- \\
29.8)\end{array}$ & $443(31.8)$ & $-20.0(-31.0,-9.0)$ & 0.000 \\
\hline Middle & $\begin{array}{l}43.1(31.9- \\
54.3)\end{array}$ & $\begin{array}{l}34.4(24.6- \\
44.1)\end{array}$ & $\begin{array}{l}37.5(23.3- \\
51.8)\end{array}$ & $\begin{array}{l}27.8(16.6- \\
39.1)\end{array}$ & $251(36.3)$ & $-15.3(-31.2,0.7)$ & 0.060 \\
\hline Rich & $\begin{array}{l}35.3(8.3- \\
62.2)\end{array}$ & $2.4(0.7-5.4)$ & $\begin{array}{l}37.4(27.8- \\
46.9)\end{array}$ & $\begin{array}{l}30.8(17.6- \\
44.1)\end{array}$ & $112(33.5)$ & $-4.5(-34.5,25.7)$ & 0.773 \\
\hline \multicolumn{8}{|l|}{ Regions } \\
\hline Tigray & $\begin{array}{l}32.7(19.8- \\
45.5)\end{array}$ & $8.2(1.8-14.6)$ & $\begin{array}{l}29.5(16.9- \\
41.9)\end{array}$ & $\begin{array}{l}12.8(2.3- \\
23.2)\end{array}$ & $40(22.5)$ & $-19.9(-36.5,-3.3)$ & 0.019 \\
\hline
\end{tabular}




\begin{tabular}{|c|c|c|c|c|c|c|c|}
\hline \multirow[t]{2}{*}{ Variables } & & 2005 & & 2016 & \multirow{2}{*}{$\begin{array}{l}2000- \\
2016 \\
n(\%)\end{array}$} & \multirow{2}{*}{$\begin{array}{l}2016-2000 \\
\text { aDiff }(95 \% \mathrm{Cl})\end{array}$} & \multirow{2}{*}{$\begin{array}{l}\text { Trend } \\
\text { p- } \\
\text { value }\end{array}$} \\
\hline & $\%(95 \% \mathrm{Cl})$ & $\%(95 \% \mathrm{Cl})$ & $\%(95 \% \mathrm{Cl})$ & $\%(95 \% \mathrm{Cl})$ & & & \\
\hline Afar & $\begin{array}{l}21.3(9.7- \\
32.8)\end{array}$ & $\begin{array}{l}24.5(10.5- \\
38.6)\end{array}$ & $\begin{array}{l}13.0(2.2- \\
23.8)\end{array}$ & $\begin{array}{l}19.7(10.7- \\
28.7)\end{array}$ & $6(20.1)$ & $-1.6(-15.7,12.7)$ & 0.834 \\
\hline Amhara & $\begin{array}{l}50.3(40.7- \\
59.8)\end{array}$ & $\begin{array}{l}33.0(22.9- \\
43.1)\end{array}$ & $\begin{array}{l}35.5(20.8- \\
50.2)\end{array}$ & $\begin{array}{l}40.4(20.6- \\
60.2)\end{array}$ & $277(40.9)$ & $-9.9(-31.9 .1,12.1)$ & 0.378 \\
\hline Oromia & $\begin{array}{l}37.7(27.9- \\
47.5)\end{array}$ & $\begin{array}{l}38.5(14.8- \\
33.4)\end{array}$ & $\begin{array}{l}32.4(22.8- \\
42.1)\end{array}$ & $\begin{array}{l}24.1(14.8- \\
33.4)\end{array}$ & 395(33.7) & $-13.6(-27.1,-0.2)$ & 0.048 \\
\hline Somali & $1.5(0.9-4.8)$ & $6.1(2.7-14.9)$ & $\begin{array}{l}8.3(0.8- \\
17.3)\end{array}$ & $0(0)$ & $3(4.2)$ & $-1.5(-4.8,1.7)$ & 0.351 \\
\hline Benishan & $\begin{array}{l}35.6(22.2- \\
48.9)\end{array}$ & $\begin{array}{l}20.9(6.0- \\
35.8)\end{array}$ & $\begin{array}{l}31.0(21.3- \\
40.7)\end{array}$ & $\begin{array}{l}29.7(12.0- \\
47.5)\end{array}$ & $10(29.9)$ & $-5.9(-28.0,16.4)$ & 0.606 \\
\hline SNNPR & $\begin{array}{l}38.0(20.7- \\
55.3)\end{array}$ & $\begin{array}{l}41.2(28.5- \\
53.9)\end{array}$ & $\begin{array}{l}29.1(13.9- \\
44.2)\end{array}$ & $\begin{array}{l}28.1(14.9- \\
41.2)\end{array}$ & 108(34.4) & $-9.9(-31.7,11.8)$ & 0.368 \\
\hline Gambela & $\begin{array}{l}40.0(21.6- \\
58.3)\end{array}$ & $\begin{array}{l}23.5(10.2- \\
36.7)\end{array}$ & $\begin{array}{l}43.3(27.2- \\
59.4)\end{array}$ & $\begin{array}{l}29.3(11.6- \\
47.0)\end{array}$ & $4(35.4)$ & $-10.7(-36.2,14.9)$ & 0.412 \\
\hline Harari & $\begin{array}{l}33.8(14.4- \\
53.1)\end{array}$ & $\begin{array}{l}22.5(13.2- \\
31.9)\end{array}$ & $\begin{array}{l}39.1(19.9- \\
58.4)\end{array}$ & $\begin{array}{l}8.0(0.1- \\
15.9)\end{array}$ & $2(25.8)$ & $-25.8(-46.7,-4.9)$ & 0.016 \\
\hline Addis Ababa & $\begin{array}{l}60.5(39.4- \\
81.5)\end{array}$ & $\begin{array}{l}30.8(10.7- \\
50.8)\end{array}$ & $\begin{array}{l}67.3(41.6- \\
92.9)\end{array}$ & $\begin{array}{l}31.9(12.2- \\
51.6)\end{array}$ & $16(47.1)$ & $-28.6(-57.4,0.3)$ & 0.052 \\
\hline Dire Dawa & $\begin{array}{l}41.1(20.4- \\
62.2)\end{array}$ & $\begin{array}{l}11.1(0.3 \\
22.5)\end{array}$ & $\begin{array}{l}30.6(11.3- \\
49.9)\end{array}$ & $\begin{array}{l}16.7(3.3- \\
30.2)\end{array}$ & $2(23.5)$ & $-24.4(-49.5,0.3)$ & 0.053 \\
\hline \multicolumn{8}{|l|}{$\begin{array}{l}\text { Regions } \\
\text { category }\end{array}$} \\
\hline Large central & $\begin{array}{l}41.9(35.9- \\
48.1)\end{array}$ & $\begin{array}{l}35.3(28.8- \\
41.8)\end{array}$ & $\begin{array}{l}32,6(25.8- \\
39.4)\end{array}$ & $\begin{array}{l}26.6(19.6- \\
33.6)\end{array}$ & $820(35.0)$ & $-15.3(-24.7,-0.6)$ & 0.001 \\
\hline $\begin{array}{l}\text { Small } \\
\text { peripheral }\end{array}$ & $\begin{array}{l}22.5(14.5- \\
30.6)\end{array}$ & $\begin{array}{l}13.8(8.1- \\
19.5)\end{array}$ & $\begin{array}{l}18.3(10.9- \\
25.5)\end{array}$ & $\begin{array}{l}9.4(5.6- \\
13.2)\end{array}$ & $23(15.6)$ & $-13.1(-22 \cdot 0,-4.3)$ & 0.004 \\
\hline Metropolis & $\begin{array}{l}54.7(38.7- \\
70.7)\end{array}$ & $\begin{array}{l}25.4(13.3- \\
37.5)\end{array}$ & $\begin{array}{l}55.7(37.2- \\
74.3)\end{array}$ & $\begin{array}{l}25.7(12.4- \\
38.9)\end{array}$ & $20(29.5)$ & $-29.0(-49.8,-8.3)$ & 0.006 \\
\hline \multicolumn{8}{|l|}{ Family size } \\
\hline $2-3$ & $\begin{array}{l}40.8(32.4- \\
49.3)\end{array}$ & $\begin{array}{l}30.4(22.4- \\
38.4)\end{array}$ & $\begin{array}{l}30.2(21.5- \\
38.9)\end{array}$ & $\begin{array}{l}25.4(15.2- \\
35.6)\end{array}$ & $340(32.1)$ & $-15.4(-28.7,-2.2)$ & 0.023 \\
\hline $4-5$ & $\begin{array}{l}44.7(35.2- \\
54.1)\end{array}$ & $\begin{array}{l}34.3(24.2- \\
44.3)\end{array}$ & $\begin{array}{l}23.5(14.2- \\
32.7)\end{array}$ & $\begin{array}{l}26.2(12.7- \\
39.7)\end{array}$ & $283(33.4)$ & $-18.5(-34.9,-2.1)$ & 0.028 \\
\hline$>5$ & $\begin{array}{l}37.7(26.0- \\
49.5)\end{array}$ & $\begin{array}{l}40.9(28.3- \\
53.5)\end{array}$ & $\begin{array}{l}47.0(33.8- \\
60.2)\end{array}$ & $\begin{array}{l}23.9(14.5- \\
33.4)\end{array}$ & $231(37.3)$ & $-13.8(-28.9,1.3)$ & 0.073 \\
\hline Overall & $\begin{array}{l}41.4(35.7- \\
47.2)\end{array}$ & $\begin{array}{l}33.9(27.9- \\
39.9)\end{array}$ & $\begin{array}{l}31.9(25.7- \\
38.3)\end{array}$ & $\begin{array}{l}25.1(18.9- \\
31.4)\end{array}$ & $863(34.0)$ & $-16.3(-24.8,-7.8)$ & 0.000 \\
\hline
\end{tabular}

Note: $n$ (\%): weighted count and proportion for each variable, \% (95\% Cl): point prevalence with its 95\% confidence interval, aDiff: indicates the point percentage change in prevalence of unintended pregnancy between 2000 to 2016 (aDiff\% 95\% Cl is the percentage point change with corresponding $95 \% \mathrm{Cl}$ of the outcome variable calculated by each of the study factors to examine the changes over the study period from 2000 to 2016), SNNPR: Southern Nations Nationalities and Peoples Region 
Table 4

Prevalence trend of unintended pregnancy by reproductive history variables among adolescents in Ethiopia, 2000-2016

\begin{tabular}{|c|c|c|c|c|c|c|c|}
\hline Variables & $2000 \mathrm{n}(\%)$ & 2005 n(\%) & 2011 n(\%) & 2016 n(\%) & $\begin{array}{l}2000-2016 \\
n(\%)\end{array}$ & $\begin{array}{l}2016-2000 \\
\text { aDiff (95\% Cl) }\end{array}$ & $\begin{array}{l}\text { Trend } \\
\text { p- } \\
\text { value }\end{array}$ \\
\hline \multicolumn{8}{|l|}{ Parity } \\
\hline 0 & $\begin{array}{l}31.7(12.6- \\
50.7)\end{array}$ & $\begin{array}{l}8.4(6.7- \\
23.5)\end{array}$ & $\begin{array}{l}53.4(11.1- \\
95.8)\end{array}$ & $\begin{array}{l}38.5(8.5- \\
85.5)\end{array}$ & $31(28.9)$ & $6.8(-4.4,57.8)$ & 0.791 \\
\hline 1 & $\begin{array}{l}38.8(31.9- \\
45.7)\end{array}$ & $\begin{array}{l}36.0(28.9- \\
43.1)\end{array}$ & $\begin{array}{l}29.4(23.2- \\
35.6)\end{array}$ & $\begin{array}{l}24.0(16.8- \\
31.1)\end{array}$ & $562(32.7)$ & $-14.9(-24.8,-4.9)$ & 0.003 \\
\hline $2-4$ & $\begin{array}{l}50.0(39.3- \\
60.7)\end{array}$ & $\begin{array}{l}33.7(21.5- \\
45.9)\end{array}$ & $\begin{array}{l}35.9(23.8- \\
47.9)\end{array}$ & $\begin{array}{l}27.1(12.3- \\
41.8)\end{array}$ & $270(37.7)$ & $-22.9(-41.2,-4.7)$ & 0.014 \\
\hline \multicolumn{8}{|l|}{$\begin{array}{l}\text { Sex of HH } \\
\text { Head }\end{array}$} \\
\hline Male & $\begin{array}{l}41.6(35.7- \\
47.5)\end{array}$ & $\begin{array}{l}33.6(27.3- \\
40.0)\end{array}$ & $\begin{array}{l}31.6(24.5- \\
38.8)\end{array}$ & $\begin{array}{l}20.6(14.1- \\
27.2)\end{array}$ & $729(33.3)$ & $-20.9(-29.8,-12.1)$ & 0.000 \\
\hline Female & $\begin{array}{l}40.0(24.2- \\
55.8)\end{array}$ & $\begin{array}{l}36.3(22.4- \\
50.1)\end{array}$ & $\begin{array}{l}33.6(21.0- \\
46.1)\end{array}$ & $\begin{array}{l}45.4(31.6- \\
59.2)\end{array}$ & $134(38.5)$ & $5.4(-15.5,26.4)$ & 0.612 \\
\hline \multicolumn{8}{|l|}{$\begin{array}{l}\text { Media } \\
\text { exposure }\end{array}$} \\
\hline No & $\begin{array}{l}36.5(30.0- \\
42.9)\end{array}$ & $\begin{array}{l}32.8(25.9- \\
39.5)\end{array}$ & $\begin{array}{l}28.8(17.6- \\
40.0)\end{array}$ & $\begin{array}{l}21.1(13.3- \\
29.0)\end{array}$ & $444(30.9)$ & $-15.4(-25.5,-5.2)$ & 0.003 \\
\hline Yes & $\begin{array}{l}51.0(40.8- \\
61.2)\end{array}$ & $\begin{array}{l}35.6(25.0- \\
46.3)\end{array}$ & $\begin{array}{l}33.8(26.5- \\
41.2)\end{array}$ & $\begin{array}{l}31.9(20.7- \\
43.1)\end{array}$ & $419(37.9)$ & $-19.1(-34.3,-3.9)$ & 0.014 \\
\hline \multicolumn{8}{|l|}{$\begin{array}{l}\text { Place of } \\
\text { delivery }\end{array}$} \\
\hline Home & $\begin{array}{l}44.4(37.7- \\
51.1)\end{array}$ & $\begin{array}{l}33.6(26.9- \\
40.3)\end{array}$ & $\begin{array}{l}35.2(27.4- \\
43.1)\end{array}$ & $\begin{array}{l}26.4(16.9- \\
35.9)\end{array}$ & $676(36.3)$ & $-18.0(-29.7,-6.3)$ & 0.003 \\
\hline $\begin{array}{l}\text { Health } \\
\text { facility }\end{array}$ & $\begin{array}{l}32.0(13.9- \\
50.2)\end{array}$ & $\begin{array}{l}43.3(23.0- \\
63.5)\end{array}$ & $\begin{array}{l}18.1(11.7- \\
35.1)\end{array}$ & $\begin{array}{l}24.9(14.5- \\
35.5)\end{array}$ & $75(26.9)$ & $-7.1(-28.0,13.9)$ & 0.511 \\
\hline \multicolumn{8}{|l|}{ Ever use FP } \\
\hline No & $\begin{array}{l}39.4(33.2- \\
45.5)\end{array}$ & $\begin{array}{l}34.8(28.7- \\
40.9)\end{array}$ & $\begin{array}{l}31.5(23.5- \\
39.6)\end{array}$ & $\begin{array}{l}24.8(17.2- \\
32.3)\end{array}$ & $666(34.1)$ & $-14.6(-24.3,-4.9)$ & 0.003 \\
\hline Yes & $\begin{array}{l}52.7(39.5- \\
65.8)\end{array}$ & $\begin{array}{l}28.1(14.0- \\
42.2)\end{array}$ & $\begin{array}{l}33.0(24.3- \\
41.7)\end{array}$ & $\begin{array}{l}25.6(15.9- \\
35.4)\end{array}$ & 197(33.6) & $-27.1(-43.4,-10.7)$ & 0.001 \\
\hline \multicolumn{8}{|l|}{$\begin{array}{l}\text { Ovulation } \\
\text { period }\end{array}$} \\
\hline Knows & $\begin{array}{l}44.6(29.7- \\
59.5)\end{array}$ & $\begin{array}{l}32.3(17.7- \\
46.9)\end{array}$ & $\begin{array}{l}26.6(10.9- \\
42.4)\end{array}$ & $\begin{array}{l}31.1(16.1- \\
45.9)\end{array}$ & 114(33.5) & $-13.5(-34.7,7.5)$ & 0.207 \\
\hline Don't know & $\begin{array}{l}41.0(34.9- \\
47.2)\end{array}$ & $\begin{array}{l}34.1(27.5- \\
40.8)\end{array}$ & $\begin{array}{l}32.9(26.6- \\
39.4)\end{array}$ & $\begin{array}{l}23.8(16.7- \\
30.8)\end{array}$ & $749(34.1)$ & $-17.2(-26.7,-7.8)$ & 0.000 \\
\hline
\end{tabular}

Note: $n$ (\%): weighted count and proportion for each variable, \% (95\% Cl): point prevalence with its 95\% confidence interval, aDiff: indicates the point percentage change in prevalence of unintended pregnancy between 2000 to 2016 ((aDiff\% 95\% Cl is the percentage point change with corresponding 95\% Cl of the outcome variable calculated by each of the study factors to examine the changes over the study period from 2000 to 2016), SNNPR: Southern Nations Nationalities and Peoples Region 


\begin{tabular}{|c|c|c|c|c|c|c|c|}
\hline Variables & $2000 \mathrm{n}(\%)$ & $2005 \mathrm{n}(\%)$ & 2011 n(\%) & 2016 n(\%) & $\begin{array}{l}2000-2016 \\
n(\%)\end{array}$ & $\begin{array}{l}2016-2000 \\
\text { aDiff (95\% Cl) }\end{array}$ & $\begin{array}{l}\text { Trend } \\
\text { p- } \\
\text { value }\end{array}$ \\
\hline \multicolumn{8}{|l|}{$\begin{array}{l}\text { Age at first } \\
\text { sex }\end{array}$} \\
\hline$<=15$ & $\begin{array}{l}46.5(36.2- \\
56.9)\end{array}$ & $\begin{array}{l}38.2(29.9- \\
46.5)\end{array}$ & $\begin{array}{l}43.4(28.7- \\
58.1)\end{array}$ & $\begin{array}{l}28.9(20.1- \\
37.7)\end{array}$ & $339(38.1)$ & $-17.6(-31.3,-4.0)$ & 0.012 \\
\hline$>15$ & $\begin{array}{l}46.2(30.1- \\
61.4)\end{array}$ & $\begin{array}{l}37.8(24.1- \\
51.4)\end{array}$ & $\begin{array}{l}41.8(20.9- \\
62.7)\end{array}$ & $\begin{array}{l}19.9(10.2- \\
29.5)\end{array}$ & $147(32.0)$ & $-26.3(-44.3,-8.4)$ & 0.004 \\
\hline \multicolumn{8}{|l|}{ Abortion } \\
\hline No & $\begin{array}{l}43.5(37.5- \\
49.5)\end{array}$ & $\begin{array}{l}35.0(28.9- \\
41.1)\end{array}$ & $\begin{array}{l}32.9(26.5- \\
39.3)\end{array}$ & $\begin{array}{l}25.1(18.9- \\
31.3)\end{array}$ & $843(34.9)$ & $-18.4(-27.1,-9.7)$ & 0.000 \\
\hline Yes & $\begin{array}{l}\text { 18.7(5.7- } \\
31.8)\end{array}$ & $0(0)$ & $\begin{array}{l}\text { 13.4(3.1- } \\
29.9)\end{array}$ & $\begin{array}{l}26.1(12.9- \\
65.1)\end{array}$ & $20(15.5)$ & $7.4(-33.9,48.5)$ & 0.727 \\
\hline \multicolumn{8}{|c|}{ ANC history } \\
\hline No & $\begin{array}{l}41.8(34.3- \\
49.4)\end{array}$ & $\begin{array}{l}35.8(28.8- \\
42.9)\end{array}$ & $\begin{array}{l}34.2(25.2- \\
43.1)\end{array}$ & $\begin{array}{l}31.3(15.0- \\
47.7)\end{array}$ & $487(37.1)$ & $-10.5(-285,7.6)$ & 0.255 \\
\hline Yes & $\begin{array}{l}47.9(37.1- \\
58.7)\end{array}$ & $\begin{array}{l}28.6(16.5- \\
40.6)\end{array}$ & $\begin{array}{l}32.9(22.5- \\
43.3)\end{array}$ & $\begin{array}{l}24.0(17.1- \\
30.9)\end{array}$ & $262(21.9)$ & $-23.9(-36.8,-11.0)$ & 0.000 \\
\hline \multicolumn{8}{|c|}{$\begin{array}{l}\text { Note: } n(\%) \text { : weighted count and proportion for each variable, } \% \text { (95\% Cl): point prevalence with its } 95 \% \text { confidence } \\
\text { interval, aDiff: indicates the point percentage change in prevalence of unintended pregnancy between } 2000 \text { to } \\
2016((\text { Diff\% } 95 \% \text { Cl is the percentage point change with corresponding } 95 \% \text { Cl of the outcome variable calculated by } \\
\text { each of the study factors to examine the changes over the study period from } 2000 \text { to 2016), SNNPR: Southern Nations } \\
\text { Nationalities and Peoples Region }\end{array}$} \\
\hline
\end{tabular}

\section{Prevalence of abortion among adolescents in Ethiopia}

Over the study period (2000-2016), the overall prevalence of abortion was 5.2\% (95\%Cl: 3.9-6.7\%). Women who had no live child had the highest prevalence of abortion (10.2\%), followed by women who resided in the metropolis regions (8.2\%) and those women who were urban residents (7.4\%). The lowest prevalence of abortion was observed among women from female headed households (1.4\%), followed by women from middle wealth households(2.7\%) (Table 5).

\section{Trends in abortion among adolescents in Ethiopia}

The prevalence of abortion was also decreased significantly from 8.3\% (95\% Cl: 5.2, 11.4\%) in 2000 to $4.1 \%$ (95\% Cl: 1.3, $6.9 \%$ ) in 2016 (Figure 1). In addition, significant reduction in percentage point of abortion was found among women with intended pregnancy (Diff $=-7.5 ; 95 \% \mathrm{Cl}:-12.9,-2.1$ ), women who had no media exposure (Diff $=-6.7 ; 95 \% \mathrm{Cl}$ : $-12.3,-1.2)$, women who had one live child (Diff $=-6.1 ; 95 \% \mathrm{Cl}:-10.9-1.2)$ and women resided in large central regions (Diff $=-4.5 ; 95 \% \mathrm{Cl}$ : $-9.1,-0.1)$ (Table 5). 
Table 5

Prevalence trend of abortion by variables among adolescents in Ethiopia, 2000-2016

\begin{tabular}{|c|c|c|c|c|c|c|c|}
\hline \multirow[t]{2}{*}{ Variables } & 2000 & 2005 & 2011 & 2016 & \multirow{2}{*}{$\begin{array}{l}2000- \\
2016 \\
n(\%)\end{array}$} & \multirow{2}{*}{$\begin{array}{l}2016-2000 \\
\text { aDiff\% (95\% } \\
\text { Cl) }\end{array}$} & \multirow{2}{*}{$\begin{array}{l}\text { Trend } \\
\text { p- } \\
\text { value }\end{array}$} \\
\hline & $\%(95 \% \mathrm{Cl})$ & $\%(95 \% \mathrm{Cl})$ & $\%(95 \% \mathrm{Cl})$ & $\%(95 \% \mathrm{Cl})$ & & & \\
\hline \multicolumn{8}{|l|}{ Age(years) } \\
\hline $15-17$ & 4.6(0.4-8.8) & $2.0(0.4-4.4)$ & $3.9(1.4-9.2)$ & $3.1(1.6-7.8)$ & $21(3.4)$ & $-1.5(-7.8,4.8)$ & 0.644 \\
\hline $18-19$ & $9.5(5.7-13.3)$ & $3.6(0.6-6.6)$ & $4.7(1.9-7.5)$ & $4.4(0.8-8.1)$ & $111(5.7)$ & $-5.0(-10.3,0.3)$ & 0.062 \\
\hline \multicolumn{8}{|l|}{ Residence } \\
\hline Urban & $5.9(1.5-13.3)$ & $\begin{array}{l}11.2(0.7- \\
21.7)\end{array}$ & $\begin{array}{l}11.1(1.4- \\
23.7)\end{array}$ & $1.2(0.7-3.1)$ & $17(7.4)$ & $-4.7(-12 \cdot 4,3 \cdot 1)$ & 0.230 \\
\hline Rural & $8.6(5.2-11.9)$ & $2.6(0.2-4.9)$ & $3.9(1.5-6.4)$ & $4.3(1.3-7.4)$ & $115(4.9)$ & $-4.2(-8.8(0.3)$ & 0.067 \\
\hline \multicolumn{8}{|l|}{ Education } \\
\hline $\begin{array}{l}\text { No } \\
\text { education }\end{array}$ & $8.1(5.2,12.3)$ & $4.0(1.8,8.7)$ & $4.3(2.1,8.8)$ & $6.6(2.1,19.5]$ & $91(5.8)$ & $-1.4(-9.6,6.8)$ & 0.732 \\
\hline $\begin{array}{l}\text { Primary and } \\
\text { above }\end{array}$ & $8.0(4.2,18.4)$ & $1.1(0.3,3.5)$ & $4.7(2 \cdot 0,10.8)$ & $2.9(1.2,6.9)$ & $41(4.1)$ & $-6.1(-13.3,1.1)$ & 0.096 \\
\hline \multicolumn{8}{|l|}{ Employment } \\
\hline $\begin{array}{l}\text { Not } \\
\text { employed }\end{array}$ & $8.3(4.3,15.6)$ & 3.2(1.3,7.5) & $4.6(2.1,9.8)]$ & 3.3(1.3,8.3) & $65(4.5)$ & $-5.0(-11.3,1.3)$ & 0.116 \\
\hline Employed & $8.5(4.7,14.9)$ & 3.3(1.1,9.9) & $3.9(1.7,8.0)$ & $5.3(1.8,14.4)$ & $54(5.7)$ & $-3 \cdot 3(-10 \cdot 7,4.1)$ & 0.385 \\
\hline \multicolumn{8}{|l|}{$\begin{array}{l}\text { Household } \\
\text { wealth }\end{array}$} \\
\hline Poor & $9.9(6.5,14.6)$ & $4.3(1.6,10.6)$ & $3.8(1.8,7.9)$ & $6.4(1.4,11.4)$ & $89(6.4)$ & $-3.4(-9.8,2.9)$ & 0.291 \\
\hline Middle & $2.6[0.5,13.4)$ & $1.1(0.2,5.2)$ & $5.7(1.8,16.9)$ & $2.6(1.3,6.5)$ & $19(2.7)$ & $-0.1(-5.9,5.9)$ & 0.991 \\
\hline Rich & $14.1(2.8,48.7)$ & $29.4(10,61.0)$ & $5.0(1.9,13.2)$ & $0.6(0.4-1.6)$ & $17(4.9)$ & $-13.5(-34.8,7.7)$ & 0.212 \\
\hline \multicolumn{8}{|l|}{$\begin{array}{l}\text { Regions } \\
\text { category }\end{array}$} \\
\hline $\begin{array}{l}\text { Large } \\
\text { central }\end{array}$ & $8.5(5.7,12.4)$ & $3.0(1.3,6.8)$ & $4.4[2.4,7.9]$ & $4.0(1.8,8.6)$ & $121(5.2)$ & $-4.5(-9.1,-0.1)$ & 0.050 \\
\hline $\begin{array}{l}\text { Small } \\
\text { peripheral }\end{array}$ & $4.1(1.7,9.7)$ & $4.1[0.5,25.1]$ & $3.6(0.7,15.4)$ & $5.8(2.7,12.2)$ & $7(4.4)$ & $1.7(-3.9,7.3)$ & 0.559 \\
\hline Metropolis & $6.3(2.1,17.4)$ & $8.7(2.8,23.6)$ & $18.4(4.3,53.2)$ & $2.2(0.8,6.5)$ & $4(8.2)$ & $-4 \cdot 1(-11 \cdot 1,3 \cdot 0)$ & 0.263 \\
\hline \multicolumn{8}{|l|}{ Family size } \\
\hline $2-3$ & $\begin{array}{l}11.5(6.3- \\
16.7)\end{array}$ & $4.3(1.0-7.7)$ & $4.8(1.0-8.6)$ & $\begin{array}{l}5.9(0.4- \\
11.9)\end{array}$ & $73(6.9)$ & $-5.5(-13.4,2.4)$ & 0.170 \\
\hline $4-5$ & $5.2(0.8-9.7)$ & $1.0(0.8-9.7)$ & $3.5(0.1-7.1)$ & $3.9(1.5-9.3)$ & $29(3.3)$ & $-1.4(-8.4,5.6)$ & 0.696 \\
\hline
\end{tabular}

Note: $n$ (\%): weighted count and proportion for each variable, \% (95\% Cl): point prevalence with its 95\% confidence interval, aDiff: indicates the point percentage change in prevalence of abortion between 2000 to 2016 (aDiff\% 95\% Cl is the percentage point change with corresponding $95 \%$ Cl of the outcome variable calculated by each of the study factors to examine the changes over the study period from 2000 to 2016), SNNPR: Southern Nations Nationalities and Peoples Region 


\begin{tabular}{|c|c|c|c|c|c|c|c|}
\hline \multirow[t]{2}{*}{ Variables } & \multirow{2}{*}{$\begin{array}{l}2000 \\
\%(95 \% \mathrm{Cl})\end{array}$} & \multirow{2}{*}{$\begin{array}{l}2005 \\
\%(95 \% \mathrm{Cl})\end{array}$} & \multirow{2}{*}{$\begin{array}{l}2011 \\
\%(95 \% \mathrm{Cl})\end{array}$} & \multirow{2}{*}{$\begin{array}{l}2016 \\
\%(95 \% \mathrm{Cl})\end{array}$} & \multirow{2}{*}{$\begin{array}{l}2000- \\
2016 \\
n(\%)\end{array}$} & \multirow{2}{*}{$\begin{array}{l}2016-2000 \\
\text { aDiff\% (95\% } \\
\mathrm{Cl})\end{array}$} & \multirow{2}{*}{$\begin{array}{l}\text { Trend } \\
\text { p- } \\
\text { value }\end{array}$} \\
\hline & & & & & & & \\
\hline$>5$ & $6.8(0.6-14.2)$ & $4.8(3.7-13.2)$ & $5.6(0.4-11.6)$ & $1.7(1.0-4.4)$ & $30(4.8)$ & $-5.1(-12.9,2.8)$ & 0.205 \\
\hline \multicolumn{8}{|l|}{ Parity } \\
\hline 0 & $\begin{array}{l}10.7(3.3- \\
24.8)\end{array}$ & $\begin{array}{l}\text { 17.6(3.1- } \\
38.1)\end{array}$ & $3.2(0.4-9.9)$ & $0.8(0.1-2.7)$ & $11(10.2)$ & $-9.9(-24.1,4.2)$ & 0.169 \\
\hline 1 & $9.7(5.9-13.6)$ & $2.4(0.6-4.2)$ & $6.1(2.7-9.4)$ & $3.6(0.6-6.7)$ & $98(5.7)$ & $-6.1(-10.9,-1.2)$ & 0.015 \\
\hline $2-4$ & $4.1(1.5-9.7)$ & $2.7(2.4-7.7)$ & $1.1(0.4-2.5)$ & $\begin{array}{l}5.9(2.8- \\
14.5)\end{array}$ & $23(3.2)$ & $1.8(-8.5,12.1)$ & 0.736 \\
\hline \multicolumn{8}{|l|}{$\begin{array}{l}\text { Sex of HH } \\
\text { Head }\end{array}$} \\
\hline Male & $8.6(5.2-11.9)$ & $3.5(0.9-6.1)$ & $5.6(2.5-8.7)$ & $4.9(1.4-8.4)$ & $127(5.8)$ & $-3.6(-8.5,1.2)$ & 0.139 \\
\hline Female & $5.7(1.8-13.2)$ & $0.7(0.6-1.9)$ & $\begin{array}{l}0.01(0.02- \\
0.07)\end{array}$ & $0.2(0.1-0.6)$ & $5(1.4)$ & $-5.5(-12.9,2.1)$ & 0.155 \\
\hline \multicolumn{8}{|l|}{$\begin{array}{l}\text { Media } \\
\text { exposure }\end{array}$} \\
\hline No & $\begin{array}{l}10.2(6.0- \\
14.4)\end{array}$ & $3.4(0.1-6.9)$ & $5.8(0.9-10.8)$ & $3.5(0.1-7.1)$ & $88(6.1)$ & $-6.7(-12.3,-1.2)$ & 0.017 \\
\hline Yes & $4.5(0.5-8.5)$ & $2.8(0.3-5.4)$ & $3.8(1.2-6.5)$ & $\begin{array}{l}5.1(0.2- \\
10.3)\end{array}$ & $44(3.9)$ & $0.6(-5.9,7.2)$ & 0.860 \\
\hline \multicolumn{8}{|l|}{$\begin{array}{l}\text { Place of } \\
\text { delivery }\end{array}$} \\
\hline Home & $7.9(4.4-11.5)$ & $3.3(0.5-6.1)$ & $3.4(0.9-5.9)$ & $5.0(0.3-9.7)$ & $93(4.9)$ & $-2.9(-8.8,2.9)$ & 0.325 \\
\hline $\begin{array}{l}\text { Health } \\
\text { facility }\end{array}$ & $\begin{array}{l}\text { 18.2(0.3- } \\
36.2)\end{array}$ & $\begin{array}{l}11.2(2.0- \\
24.3)\end{array}$ & $\begin{array}{l}11.2(3.8- \\
26.3)\end{array}$ & $1.8(0.9-4.5)$ & $21(7.4)$ & $-16.5(-34.7,1.7)$ & 0.076 \\
\hline \multicolumn{8}{|l|}{ Ever use FP } \\
\hline No & $8.6(5.2-12.0)$ & $3.0(0.4-5.6)$ & $4.5(1.5-7.5)$ & $3.9(0.7-7.2)$ & $103(5.3)$ & $-4.6(-9.4,0.1)$ & 0.054 \\
\hline Yes & $6.5(0.4-13.4)$ & $4.4(0.6-9.3)$ & $4.7(0.2-9.2)$ & $4.2(1.1-9.5)$ & $29(4.8)$ & $-2.3(-11.0,6.4)$ & 0.606 \\
\hline \multicolumn{8}{|l|}{$\begin{array}{l}\text { Pregnancy } \\
\text { plan }\end{array}$} \\
\hline Intended & $\begin{array}{l}11.5(6.9- \\
16.1)\end{array}$ & $4.9(1.4-8.3)$ & $5.8(2.4-9.2)$ & $4.0(1.2-6.8)$ & $111(6.6)$ & $-7.5(-12.9,-2.1)$ & 0.007 \\
\hline Unintended & $3.7(0.9-6.6)$ & 0 & $1.9(1.0 .5-4.3)$ & $\begin{array}{l}4.2(3.4- \\
11.9)\end{array}$ & $21(2.4)$ & $0.5(-7.7,8.7)$ & 0.909 \\
\hline \multicolumn{8}{|l|}{ ANC history } \\
\hline No & $8.1(3.9-12.2)$ & $4.6(1.0-8.2))$ & $3.2(0.7-5.7)$ & $\begin{array}{l}5.4(3.7- \\
14.5)\end{array}$ & $72(5.5)$ & $-2.7(-12.7,7.3)$ & 0.595 \\
\hline
\end{tabular}

Note: $n$ (\%): weighted count and proportion for each variable, \% (95\% Cl): point prevalence with its $95 \%$ confidence interval, aDiff: indicates the point percentage change in prevalence of abortion between 2000 to 2016 (aDiff\% 95\% Cl is the percentage point change with corresponding $95 \%$ Cl of the outcome variable calculated by each of the study factors to examine the changes over the study period from 2000 to 2016), SNNPR: Southern Nations Nationalities and Peoples Region 


\begin{tabular}{|c|c|c|c|c|c|c|c|}
\hline \multirow[t]{2}{*}{ Variables } & 2000 & 2005 & 2011 & 2016 & \multirow{2}{*}{$\begin{array}{l}2000- \\
2016 \\
n(\%)\end{array}$} & \multirow{2}{*}{$\begin{array}{l}2016-2000 \\
\text { aDiff\% }(95 \% \\
\text { Cl) }\end{array}$} & \multirow{2}{*}{$\begin{array}{l}\text { Trend } \\
\text { p- } \\
\text { value }\end{array}$} \\
\hline & $\%(95 \% \mathrm{Cl})$ & $\%(95 \% \mathrm{Cl})$ & $\%(95 \% \mathrm{Cl})$ & $\%(95 \% \mathrm{Cl})$ & & & \\
\hline Yes & $\begin{array}{l}10.9(3.3- \\
18.6)\end{array}$ & $1.0(0.5-2.4)$ & $5.7(0.4-10.9)$ & $3.2(0.4-6.0)$ & $41(4.9)$ & $-7.7(-15.9,0.4)$ & 0.064 \\
\hline Overall & 8.3(5.2-11.4) & $3.2(0.9-5.5)$ & 4.6(2.1-7.0) & 4.1(1.3-6.9) & $\begin{array}{l}5.2(3.9- \\
6.7)\end{array}$ & $-4.2(-8.4-0.03)$ & 0.048 \\
\hline \multicolumn{8}{|c|}{$\begin{array}{l}\text { Note: } n(\%) \text { : weighted count and proportion for each variable, } \% \text { (95\% Cl): point prevalence with its } 95 \% \text { confidence } \\
\text { interval, aDiff: indicates the point percentage change in prevalence of abortion between } 2000 \text { to } 2016 \text { (aDiff\% } 95 \% \text { Cl is } \\
\text { the percentage point change with corresponding } 95 \% \text { Cl of the outcome variable calculated by each of the study factors } \\
\text { to examine the changes over the study period from } 2000 \text { to 2016), SNNPR: Southern Nations Nationalities and Peoples } \\
\text { Region }\end{array}$} \\
\hline
\end{tabular}

\section{Factors associated with unintended pregnancy among adolescents in Ethiopia}

In the null model, about $35.6 \%$ of the total variation on unintended pregnancy was occurred at the community level which is accounted to the community-level factors (ICC=0.356). The variation of unintended pregnancy between clusters suggested to conduct multilevel analyses. The model fitness comparison was carriedout using information criteria (IC) and the model with the lowest IC value (Model 4) was the best-fitted model (Suplemetary Table 1). In the multivariable multilevel logistic regression analysis, age, marital status, parity, and regions were factors significantly associated with unintended pregnancy among adolescents aged from 15-19 years old in Ethiopia. Adolescents aged above 17 years old had lower odds of having unintended pregnancy compared to those aged $15-17$ years old ( $\mathrm{AOR}=0.30 ; 95 \% \mathrm{Cl}$ : 0.17-0.51). Married women had significantly lower odds of having untended pregnancy compared to those who were unmarried (AOR=0.14; $95 \% \mathrm{Cl}$ : 0.070.28). Women who had two and more live children had significantly higher odds of unintended pregnancy compared with their counter parts (AOR=1.68; 95\% Cl: 1.02-2.75). In addition, women who resided in large central (AOR=3.32; 95\%Cl: $1.86-$ 5.63), and metropolis (AOR=3.12; $95 \% \mathrm{Cl}: 1.20-8.12)$ regions had significantly higher rate of having unintended pregnancy compared to those who resided in the small peripheral region (Table 6). 
Table 6

Multilevel logistic regression analysis of individual and community-level factors associated with unintended pregnancy among adolescents in Ethiopia

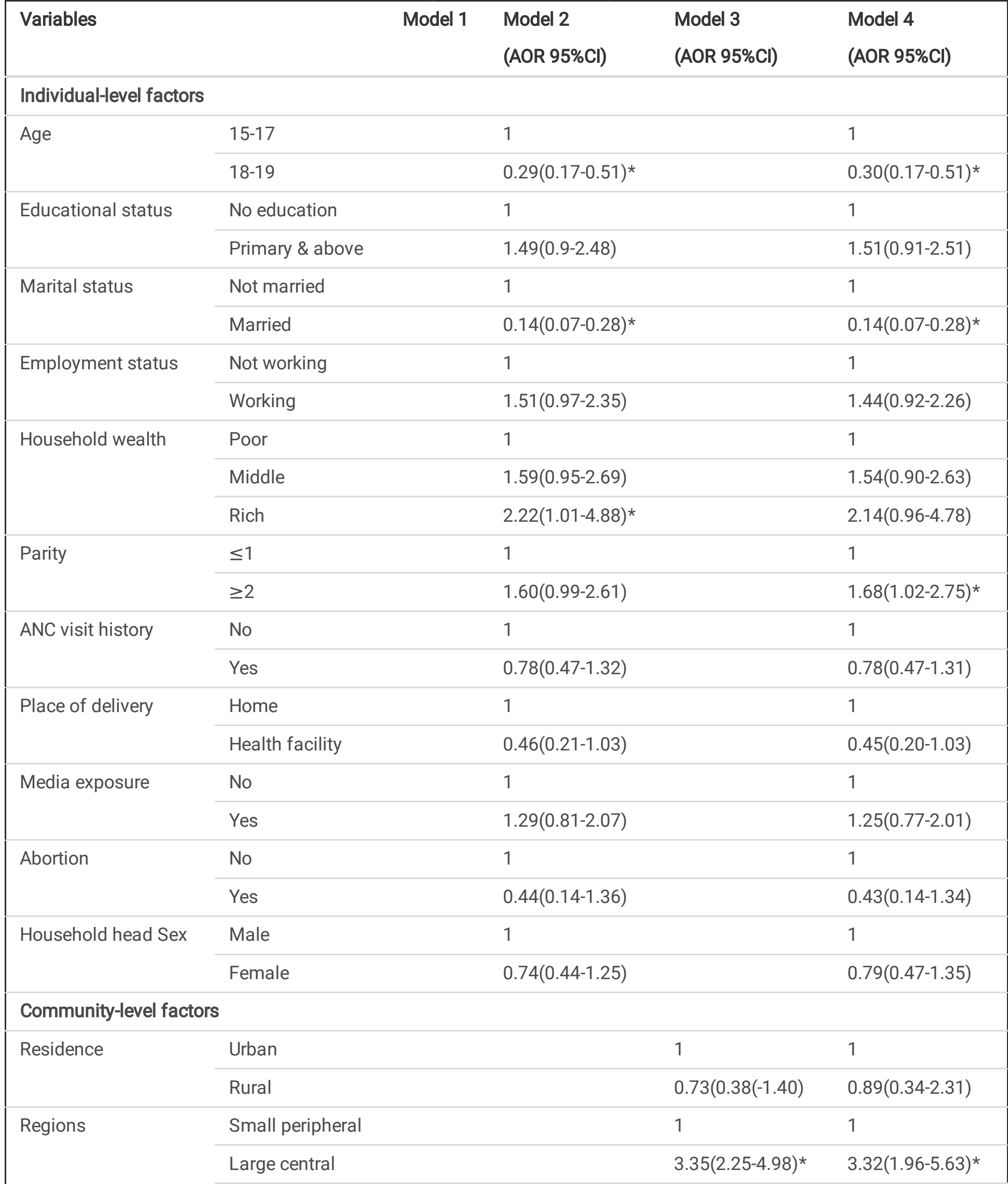

Note: Variables with $p$-value $<0.25$ in bivariable multilevel logistic regression analysis were included in multivariable analysis to control confounders. * significant at $p$-value $<0.05$ 


\begin{tabular}{|c|c|c|c|c|}
\hline Variables & Model 1 & $\begin{array}{l}\text { Model } 2 \\
\text { (AOR 95\%Cl) }\end{array}$ & $\begin{array}{l}\text { Model } 3 \\
\text { (AOR 95\%Cl) }\end{array}$ & $\begin{array}{l}\text { Model } 4 \\
\text { (AOR 95\%Cl) }\end{array}$ \\
\hline Metropolis & & & $3.48(1.72-7.04)^{*}$ & $3.12(1.20-8.12)^{*}$ \\
\hline
\end{tabular}

\section{Factors associated with abortion among adolescents in Ethiopia}

The null model showed that $57.8 \%$ of the total abortion variations resulted from the variabilities among clusters and the rest from individual differences (ICC=0.578). The clustering effect indicated to conduct multilevel analyses and Model 4 was the best-fitted model with lowest IC value (Suplemetary Table 1). In the multivariable multilevel logistic regression analysis, household wealth status, pregnancy plan status, family size, sex of household head and residence were factors significantly associated with abortion among adolescents aged from 15-19 years old in Ethiopia. Adolescents from middle wealth households (AOR=0.14; 95\% Cl: 0.03-0.64) and rich wealth households (AOR=0.09; 95\% Cl: 0.01-0.74) had significantly lower rate of abortion compared to those adolescents from poor wealth households. The odds of abortion among adolescents who had unintended pregnancy was significantly lower than those who had intended pregnancy (AOR= 0.23; 95\% Cl: 0.08-0.67). Likewise, adolescent girls from female headed households had significantly lesser rate of abortion compared to those adolescent girls from male headed households ( $\mathrm{AOR}=0.11 ; 95 \% \mathrm{Cl}$ : 0.01-0.91). Adolescents from rural areas also had lower rate of abortion compared to urban counterparts (AOR=0.08; 95\%Cl: 0.01-0.45) (Table 7). 
Table 7

Multilevel logistic regression analysis of individual and community-level factors associated with abortion among adolescents in Ethiopia

\begin{tabular}{|c|c|c|c|c|c|}
\hline Variables & & Model 1 & $\begin{array}{l}\text { Model } 2 \\
\text { (AOR 95\%Cl) }\end{array}$ & $\begin{array}{l}\text { Model } 3 \\
\text { (AOR 95\%Cl) }\end{array}$ & $\begin{array}{l}\text { Model } 4 \\
\text { (AOR 95\%Cl) }\end{array}$ \\
\hline \multicolumn{6}{|l|}{ Individual-level factors } \\
\hline \multirow[t]{2}{*}{ Age } & $15-17$ & & 1 & & 1 \\
\hline & $18-19$ & & $1.06(0.32-3.46)$ & & $1.07(0.32-3.51)$ \\
\hline \multirow[t]{2}{*}{ Educational status } & No education & & 1 & & 1 \\
\hline & Primary \& above & & $0.68(0.20-2.30)$ & & $0.70(0.20-2.45)$ \\
\hline \multirow[t]{2}{*}{ Marital status } & Not married & & 1 & & 1 \\
\hline & Married & & $0.52(0.07-3.91)$ & & $0.55(0.07-4.17)$ \\
\hline \multirow[t]{3}{*}{ Household wealth } & Poor & & 1 & & 1 \\
\hline & Middle & & $0.17(0.04-0.74)^{\star}$ & & $0.14(0.03-0.64)^{*}$ \\
\hline & Rich & & $0.35(0.16-1.97)$ & & $0.09(0.01-0.74)^{\star}$ \\
\hline \multirow[t]{2}{*}{ Parity } & $\leq 1$ & & 1 & & 1 \\
\hline & $\geq 2$ & & $0.71(0.15-3.29)$ & & $0.75(0.16-3.56)$ \\
\hline \multirow[t]{2}{*}{ Place of delivery } & Home & & 1 & & 1 \\
\hline & Health facility & & $1.84(0.42-8.08)$ & & $1.29(0.24-7.07)$ \\
\hline \multirow[t]{2}{*}{ Family size } & $\leq 3$ & & 1 & & 1 \\
\hline & $\geq 4$ & & $0.21(0.06-0.79)^{\star}$ & & $0.20(0.05-0.75)^{*}$ \\
\hline \multirow[t]{2}{*}{ Pregnancy plan } & Intended & & 1 & & 1 \\
\hline & Unintended & & $0.25(0.08-0.74)^{\star}$ & & $0.23(0.08-0.67)$ * \\
\hline \multirow[t]{2}{*}{ Media exposure } & No & & 1 & & 1 \\
\hline & Yes & & $2.22(0.64-7.68)$ & & $1.82(0.48-6.85)$ \\
\hline \multirow[t]{2}{*}{ Sex of household head } & Male & & 1 & & 1 \\
\hline & Female & & $0.11(0.01-0.83)^{\star}$ & & $0.11(0.01-0.91)$ * \\
\hline \multicolumn{6}{|l|}{ Community-level factors } \\
\hline \multirow[t]{2}{*}{ Residence } & Urban & & & 1 & 1 \\
\hline & Rural & & & $0.57(0.18-1.86)$ & $0.08(0.01-0.45)^{*}$ \\
\hline \multirow[t]{3}{*}{ Regions } & Small peripheral & & & 1 & 1 \\
\hline & Large central & & & $1.25(0.49-3.14)$ & $2.31(0.54-9.96)$ \\
\hline & Metropolis & & & $2.18(0.50-9.56)$ & $2.58(0.26-25.9)$ \\
\hline
\end{tabular}




\section{Discussion}

This study assessed trend of unintended pregnancy, abortion and associated factors among adolescents in Ethiopia from 2000 to 2016 . The overall prevalence of unintended pregnancy was $34.0 \%$ among adolescents in Ethiopia. Studies done in sub-Saharan Africa countries including Ethiopia also showed that unintended pregnancy rate in sub-Saharan Africa remains high, especially among adolescents $[4,28,29]$. The prevalence of unintended pregnancy among Ethiopian adolescents decreased significantly from $41.4 \%$ in 2000 to $25.1 \%$ in 2016 , potentially reflecting the impacts of the national reproductive health interventions in Ethiopia [24]. Factors associated with a lower likelihood of unintended pregnancy among adolescent girls in Ethiopia included being aged above 17 years old and being married. While, higher parity, resided in large central and metropolis regions were significantly associated with higher odds of untended pregnancy.

The prevalence of abortion also decreased significantly from $8.3 \%$ in 2000 to $4.1 \%$ in 2016 . Belonging to middle and rich wealth households, having unintended pregnancy, female head households and being rural residents were factors significantly associated with lower likelihood of abortion among adolescents in Ethiopia. These reduction may be attributable to the relatively high maternal health service utilization like modern contraceptive utilization (35\%) ) in 2016 [24] compared to (6\%) in 2000 [30]. These findings reflected that reproductive health interventions targeting the reduction of unintended pregnancies and induced abortion among adolescents should be strengthen more in order to reduce unintended pregnancies, abortion related mortality and morbidity, improve adolescent health and permitting adolescents to attain their economic potential [5, 31-34].

This study showed that adolescent girls aged below 18 years old had higher odds of having unintended pregnancy. Similarly, studies conducted in Africa indicated that the risk of unplanned pregnancies decreases with age that older mothers had lower odds of unintended pregnancy in developing countries [35-40]. This might be due to older women had relatively better knowledge on contraceptive methods to prevent unintended pregnancy and lower contraceptive failure rate $[35,41]$. Moreover, as they are getting older, women might also become more literate about the importance and accessibility of reproductive or maternal health services. In addition, this could be also as the results of older women are less likely to engage in risky sexual behaviors such as unprotected sexual intercourse and sex under the influence of drinking alcohol[42, 43]. However, other literatures revealed a negative relationship between age and unintended pregnancy[37, 44, 45]. This finding might be related to the fact that adult women might already have the desirable number of children and considered any additional pregnancy as mistimed or unwanted.

In this study, education was no significantly associated with untended pregnancy. There was no agreement across researches regarding the impact of the level of education on the risk of having unintended pregnancies. A systematic review on prevalence and determinants of unintended pregnancy in Sub-Saharan Africa revealed that the outcomes ranged from reporting no association with level of education and odds of having unintended pregnancies in Nigeria[46], South Africa[47], Ethiopia[48], and Kenya[39], through an increased risk of reporting the last pregnancy as unintended as the level of education increases in the Democratic Republic of Congo and Ethiopia [49,50], to an increase in the level of education being protective against unintended pregnancies in Nigeria[51], in Tanzania[52] and in Ethiopia[53]. Researches that analyzed demographic health survey data also found discordant findings in Ethiopia [23]; in the study by Tebekaw, education was protective against unintended pregnancies [23], while the other study found no association between education and unintended pregnancy. A study done in Nairobi also found that education was protective against having an unintended pregnancy, with a $10 \%$ decrease in the risk of having an unintended pregnancy [53].

In this study, married women had significantly lower odds of untended pregnancy compared to those who were unmarried. This find is consistent with studies conducted in Ethiopia [6, 29, 54-56], in Kenya[39] and in South Africa[57]; reported that unmarried mothers were at higher odds of unintended pregnancy. The potential reason is that unmarried adolescent girls may unintentionally participate in sexual activity and this is most likely unwanted if the pregnancy is occurred. Additionally, our culture and community might cause a significant influence on unmarried women not to use contraceptive because of

Page 20/28 
sex is not recommended before the women became married. In addition, this could be due to the fact that married mothers are less likely to engage in risky sexual behaviors such as unprotected sexual intercourse and sex under the influence of drinking alcohol [42-44]. On the other hand, unmarred adolescent girls could engage in sexual activity for pleasure. Therefore, if pregnancy occurs it is more likely to be unintended. Furthermore, they are less likely to use contraceptive methods $[56,58]$. Other reasons could be difficulty in accessing contraception, not having adequate financial and social support to provide for their unborn child [58].

The study also indicated that adolescent mothers who had relatively higher parity experienced more risk of having unintended pregnancy. This finding is supported by researches done in Ethiopia[31, 56, 59], in Ghana[45] and in Pakistan [35], that reported multiparous and grand multiparous mothers had higher odds of unintended pregnancy compared with primiparou women. Similarly, studies from Brazil and USA have reported that mothers who have more alive children are more likely to experience an unintended pregnancy [60]. The possible explanation might be high parity woman might already have adequate children with a decreasing intention for the next pregnancy. This might be also due to mothers with higher parity might be busy in caring their children and family and this affects their getting of information, accessing and utilization of maternal health services such as contraceptive methods which in turn end up with unintended pregnancy. In addition, it might imply the gaps in provision of contraceptives counseling and education [4].

Evidence has shown that prevalence of unintended pregnancy differs across socioeconomic levels at national and subnational levels $[4,28,29]$. This study also found significant variations of unintended pregnancy rate across regions in Ethiopia that adolescent girls from large central and metropolitan regions had higher likelihood of unintended pregnancy. This regional difference of unintended pregnancy prevalence is congruent with research findings from Ethiopia [23, 27], Kenya [39], and Ghana [45]. This could be due to pregnancies might be appreciated and accepted in small peripheral regions and mothers in this region did not think about unintended pregnancy. Nevertheless, women in metropolitan and large central regions may be busy because of their intention to advance their socio-economic status and mostly their pregnancies are more likely to be unintended $[28,29]$. These variations a cross regions reflect the significance of disaggregated data for evidence-based policymaking and program design and context-specific interventions are required in order to reduce unintended pregnancy and associated abortion among adolescents in Ethiopia.

Adolescents from middle and rich wealth households had significantly lower rate of abortion compared to those from poor wealth households. This finding is supported by previous researches evidence that women with lower income had higher rate of induced abortion than those with higher income [61, 62]. Finding from the EDHS Surveys have shown that modern contraception use increases sharply with wealth, ranging from $20 \%$ in the lowest wealth index to $47 \%$ in the highest wealth index quintile[24]. Hence, the low contraceptive utilization among adolescents with lower economic status may account for the higher odds of abortion [63]. It might also due to financially not prepared to raise their unborn child. Therefore, women in poor wealth households may get an induced abortion in order to limit births due to economic, demographic and social reasons [64]. Abortion was also less likely to occur among women whose husband had occupation [45, 67]. This might be because employed husbands have a positive attitude in avoiding pregnancy termination by increasing their economic status and women might get frequent health institution visits and health information about consequences of pregnancy termination. Adolescents from poor income families may be also more risky to sexual coercion and rape that can lead to unintended pregnancy and induced abortion [59] .

Furthermore, adolescents from female headed households experienced significantly lower rate of abortion compared to those from male headed households. Likewise, previous studies also documented that female-headed households were less likely to experience unwanted adolescent pregnancy $[4,51]$. This might be due to adolescent girls from female headed households would have access to information where to get the services and perhaps may be they had their own income to raise their child, as a result women might get frequent health institution contact for antenatal care, delivery and health information about consequences of pregnancy termination. And it could be also due to relatively better level of reproductive 
health knowledge and access to family planning services, freedom of decision making on reproductive matters among female head households compared with male headed household counterparts $[45,66]$.

In this study, the likelihood of abortion among women who had unintended pregnancy was significantly lower than women who had intended pregnancy. A study conducted in West Arsi Zone, Ethiopia also showed a negative relationship between abortion history and unintended pregnancy [56]. Possible explanation may be even though women who had unintended pregnancy tend to avoid unwanted pregnancies by inducing their current pregnancy, they might still lack the self-rule to make decisions about their pregnancy intention. As it is the case with many other areas of reproductive health, husbands appear to be the primary decision makers with consider to pregnancy [67]. Moreover, studies also indicated that previous unintended pregnancies and previous abortions were reported to be risk factors for subsequent unintended pregnancies [68]. This could be an indication of inadequate sex education, contraception education, or sub-optimal post-abortion counseling among women with previous unintended pregnancies

Place of residence was also a significant factor associated with abortion. Adolescents from rural areas also had lower rate of abortion compared to urban counterparts. This finding was supported by studies conducted in Ethiopia[69].This might be because adolescents from urban areas are exposed to different factors that make them risky to sexual behaviors that will end in unwanted pregnancy and induced abortion. Urban girls have a lot of access to addiction of stimulants and alcohol than rural girls as well as better access to abortion services. Moreover, adolescents from rural areas might also fear to induce abortion believing that terminating pregnancy as a sin due to their religion believes [24].

\section{Strengths And Limitations Of The Study}

The research is based on nationally representative data with large sample size so that the observed findings more likely to show the status of unintended pregnancy and abortion among adolescents in Ethiopia. The study has the potential to provide evidence for policy-makers and program planners to design appropriate intervention strategies both at national and regional levels because the estimates are based on the national survey data. Nevertheless, the study had limitations because of the Ethiopian demographic and health surveys data are mostly based on respondents' self-report and could have the possibility of recall bias. In addition, it is difficult to establish a cause-effect relationship between the outcomes and independent variables due to the research is based on cross-sectional data.

\section{Conclusion}

The result showed that the prevalence of unintended pregnancy among adolescents in Ethiopia significantly decreased from $41.4 \%$ in 2000 to $25.1 \%$ in 2016 . Despite this reduction, approximately one-fourth of pregnancies were unintended among adolescents in Ethiopia, indicating still the magnitude of the problem is high. This finding suggested that unintended pregnancy is still one of the .major reproductive health challenges faced by adolescents in Ethiopia. Factors associated with a lower likelihood of unintended pregnancy among adolescents included being aged above 17 years old and being married. While, higher parity, resided in large central and metropolis regions were significantly associated with higher odds of untended pregnancy. Similarly, the prevalence of abortion was also decreased significantly from $8.3 \%$ in 2000 to $4.1 \%$ in 2016. However, abortion is still one of the common reproductive health problems among Ethiopian adolescent girls. Belonging to middle and rich wealth households, having unintended pregnancy, being rural resident and female head households were factors significantly associated with lower likelihood of abortion among adolescents in Ethiopia. Thus, providing maternal and child services along with adequate sex education, contraception education, and health information about consequences of pregnancy termination would be vital. Moreover, those identified high-risk groups deserve special attention in terms of improving adolescents' reproductive health knowledge and access to FP services, enhancing decision making autonomy on reproductive matters including increasing accessibility and availability of quality maternal health services.

Page 22/28 


\section{Abbreviations}

CAC

Comprehensive Abortion Care

CSA

Central Statistics Agency

DHS

Demographic and Health Survey

EDHS

Ethiopia Demographic and Health Survey

HAPCO

HIV/AIDS Prevention and Control Office

MDG

Millennium Development Goals

PHCCO

Population and Housing Census commission Office

$\mathrm{SRH}$

Sexual and Reproductive Health

\section{Declarations}

\section{Ethics approval and consent to participate}

Ethical clearance for the 2016 EDHS was provided by the Ethiopian Health and Nutrition Research Institute (EHNRI) Review Board, the National Research Ethics Review Committee (NRERC) at the Ministry of Science and Technology, the Institutional Review Board of ICF International, and the communicable disease control (CDC). We confirm that the study was conducted in accordance with the Helsinki Declaration. Written consent was obtained from each participant. In addition, parents/legal guardians were conscented for minors aged 15 and 16 years. The parental conscent for minors was also approved by the above mentioned national and international ethical bodies. However, the dataset of the 2016 EDHS is not available as a public domain survey dataset. The author requested access to the data from demographic, health survey program team and access was granted to use the data for this research.

\section{Consent for publication}

Not applicable

\section{Availability of data and materials}

The data sets used and/or analyzed during the current study are available in the Ethiopian statistical agency and ministry of health.

\section{Competing interests}

The authors declare that they have no competing interests.

\section{Funding}

We have no funding for this research

\section{Authors' contributions}


TA conceived the study ideas, design and analyzed data; TS wrote the draft manuscript; FT and TS participated in the study design, edited the manuscript and contributed to the final analysis. All authors read and approved the final manuscript.

\section{Acknowledgements}

The authors would like to acknowledge that the Ethiopian Demographic and Health Survey 2016 data used in this study were obtained from the DHS office, they have given a permission to access the data, after we have prepared the proposal on the title.

\section{References}

1. Heilman J.M, Kemmann E, Bonert M, Chatterjee A, Ragar B, Graham M. et al. A key tool for global public health promotion. Journal of Medical Internet Research, 2011. 13(1): e14.

2. Alvey J, Bryant A, Curtis S, Speizer I, Morgan P, Tippett R. et al. Trends in Abortion Incidence and Availability in North Carolina, 1980-2013. South Medical Journal, 2017. 110(11):714-721.

3. Santelli J, Rochat R, Gilbert B, Curtis K, Cabral R, Schieve L. et al. The measurement and meaning of unintended pregnancy. Perspectives on Sexual and Reproductive Health. 2003: 35(2):94-101.

4. Bain LE, Marjolein BM, BuningTC. Prevalence and Determinants of Unintended Pregnancy in Sub -Saharan Africa: A Systematic Review. Africa Journal Reproductive Health 2020; 24(2):187-205.

5. World Health Organization. Unsafe abortion: global and regional estimates of the incidence of unsafe abortion and associated mortality in 2008. 6th ed. Geneva: World Health Organization; 2011.

6. Getu MK, Hailu M, Berta M, Fekadu M. Unintended Pregnancy in Ethiopia: Community Based Cross-Sectional Study. Obstetrics and Gynecology International , 2016; 5:4374791.

7. Bearak J, Popinchalk A, Alkema L, Sedgh G . Global, regional, and subregional trends in unintended pregnancy and its outcomes from 1990 to 2014: estimates from a Bayesian hierarchical model. Lancet Glob Health, 2018. 6(4): e380e389.

8. Calvert C, Owolabi O, Yeung F, Pittrof R, Ganatra B,Tunçalp O. et al. The magnitude and severity of abortion-related morbidity in settings with limited access to abortion services: a systematic review and meta-regression. BMJ Global Health, 2018. 3(3):e000692.

9. Sedgh, G, Bearak J, Singh S, Bankole A, Popinchalk A, Ganatra B. et al. Abortion incidence between 1990 and 2014 : global, regional, and subregional levels and trends. Lancet, 2016. 388(10041):258-67.

10. Paxman JM. Reproductive health, youth, and the law. WHO Chronicle, 1984. 38(5):199-207.

11. Salam RA, Das JK, Lassi ZS, BHutta ZA. Adolescent health interventions:Evidence gaps and research priorities. Journal of Adolescent Health. 2016.59:S88eS92.

12. Ganchimeg T, Ota E, Morisaki N,Laopaiboon M, Lumbiganon P,Zhang J. et al. Pregnancy and childbirth outcomes among adolescent mothers: a World Health Organization multicountry study. BJOG: An International Journal of Obstetrics \& Gynaecology, 2014. 121: 40-48.

13. Adhikari R. Factors affecting awareness of emergency contraception among college students in Kathmandu, Nepal. BMC Womens Health. 2009. 9:27.

14. Moore AM, Gebrehiwot Y, Fetters T, Wado T, Bankole A, Singh S. et al. The Estimated Incidence of Induced Abortion in Ethiopia, 2014: Changes in the Provision of Services Since 2008. International Perspect Sex Reproducative Health, 2016. 42(3):111-120.

15. Nojomi M, Akbarian A, and Ashory-Moghadam S. Burden of abortion: induced and spontaneous. Archives Iranian Medical journal, 2006. 9(1): 39-45. 
16. Orner P.J, Bruyn M, Barbosa R, Boonstra H, Gatsi-Mallet J, Cooper D. Access to safe abortion: building choices for women living with HIV and AIDS. Journal of the International AIDS Society, 2011.14:54.

17. Finer L.B. and Henshaw S.K. Disparities in rates of unintended pregnancy in the United States, 1994 and 2001. Perspectives on sexual and reproductive health, 2006. 38(2):90-96.

18. Cameron S, Glasier A, Lohr P.A, Moreau C, Munk-Olsen T, Oppengaard K.S. et al. Induced abortion. Human Reproduction, 2017. 32(6): 1160-1169.

19. Polis C.B, Mhango C, Philbin J, Chimwaza W, Chipeta E, Msusa A. Incidence of induced abortion in Malawi, 2015. PLoS One, 2017. 12(4):e0173639.

20. Bankole A, Adewole I, Hussain R, Awolude O, Singh S, Akinyemi J.The Incidence of Abortion in Nigeria. International Perspect Sex Reprod Health, 2015. 41(4):170-81.

21. Chae S, Kayembe P, Philbin J, Mabika C, Bankole A. The incidence of induced abortion in Kinshasa, Democratic Republic of Congo, 2016. PLoS One, 2017. 12(10): e0184389.

22. Singh S, Fetters T, Gebreselassie H, Abdella A, Gebrehiwot $Y$, Kumbi S, et al. The estimated incidence of induced abortion in Ethiopia, 2008. International Perspect Sex Reproductive Health, 2010. 36(1):16-25.

23. Tebekaw Y, Aemro B, andTeller C. Prevalence and determinants of unintended childbirth in Ethiopia. BMC Pregnancy Childbirth, 2014. 14:326.

24. Central Statistical Agency (CSA) Ethiopia and ICF. Ethiopia Demographic and Health Survey 2016. Addis Ababa: CSA and ICF; 2016.

25. Bongaarts J, Mauldin W.P, and Phillips J.F. The demographic impact of family planning programs. Studies in family planning, 1990. 21(6):299-310.

26. Central Statistical Agency (CSA) Ethiopia and ICF. Ethiopia Demographic and Health Survey, 2005. Central Statistical Authority.2006.

27. Teshale AB. and Tesema GA. Magnitude and associated factors of unintended pregnancy in Ethiopia: a multilevel analysis using 2016 EDHS data. BMC Pregnancy and Childbirth (2020) 20:329.

28. Ameyaw EK, BuduE, Sambah F, Baatiema L, Appiah F, Seidu A-A, et al. Prevalence and determinants of unintendedpregnancy in subSaharan Africa: A multi-country analysis of demographic and healthsurveys. PLoS ONE 2019.14(8): e0220970.

29. Alene M, Yismaw L, Berelie Y, Kassie B, YeshambelR, Assemie MA. Prevalenceand determinants of unintended pregnancy in Ethiopia: A systematic review and meta-analysis of observational studies.PLoS ONE . 2020.15(4): e0231012.

30. Central Statistical Agency (CSA) Ethiopia and ICF. Ethiopia Demographic and Health Survey 2000. Addis Ababa: CSA and ICF; 2001.

31. Gerdts C, Dobkin L, Foster DG and Schwarz EB. Side Effects, Physical Health Consequences, and Mortality Associated with Abortion and Birth after an Unwanted Pregnancy. Women's Health Issues. 2016 Jan 1;26(1):55-9. .

32. Singh A, Singh A and Thapa S. Adverse consequences of unintended pregnancy for maternal and child health in Nepal. Asia Pac J Public Health. 2015;27(2):1481-1491.

33. Finer LB and Sonfield A. The evidence mounts on the benefits of preventing unintended pregnancy. Contraception. 2013;87(2):126-7.

34. United Nations. Sustainable development goals - United Nations. United Nations Sustainable Development goals. 2015.

35. Habib A, Greenow CR. Prevalence and determinants of unintended pregnancies amongst women attending antenatal clinics in Pakistan BMC Pregnancy and Childbirth. 2017;17:156. 
36. Yaya S, Amouzou A, Uthman OA, Ekholuenetale M, Bishwajit G, Udenigwe O, et al. Prevalence and determinants of terminated and unintended pregnancies among married women : analysis of pooled cross-sectional surveys in Nigeria. 2018. BMJ Glob Health 2018;3:e000707.

37. Adeniyi OV, Ajayi Al, Moyaki MG, Ter Goon D, Avramovic G, Lambert J. High rate of unplanned pregnancy in the context of integrated family planning and HIV care services in South Africa. BMC Health Services Research.2018; 18:140.

38. Martin E. Prevalence and Determinants of Unintended Pregnancies in Malawi. African Population Studies. 2014;28(1):551-63.

39. Ikamari L, Izugbara C, Ochako R. Prevalence and determinants of unintended pregnancy among women in Nairobi , Kenya. BMC pregnancy and childbirth. 2013;13:1-9.

40. Rizvi F, Williams J, Hoban E. Factors influencing unintended pregnancies amongst adolescent girls and young women in Cambodia. Int. J. Environ. Research Public Health 2019;16:400.

41. Exavery A, Kanté AM, Njozi M, Tani K, Doctor HV, Hingora A, et al. Predictors of mistimed, and unwanted pregnancies among women of childbearing age in Rufiji , Kilombero, and Ulanga districts of Tanzania. Reproductive Health. 2014; 11:63.

42. Tadesse G, Yakob B. Risky sexual behaviors among female youth in Tiss Abay, a semi-urban area of the Amhara Region, Ethiopia. PLoS One. 2015; 10(3).

43. Taylor-Seehafer M, Rew L. Sexual behavior among adolescent women. JSPN. 2000;5(1):15-25.

44. Ameyaw EK. Prevalence and correlates of unintended pregnancy in Ghana: Analysis of 2014 Ghana Demographic and Health Survey. Maternal Health, Neonatology Perinatology. 2018; 4(1):17.

45. Nyarko SH. Unintended Pregnancy among Pregnant Women in Ghana: Prevalence and Predictors. Journal of Pregnancy. 2019. 8:2920491.

46. Agida TE, Akaba GO, Ekele BA and Adebayo F. Unintended pregnancy among antenatal women in a tertiary hospital in North Central Nigeria. Niger Medical Journal. 2016;57(6):334-8.

47. Christofides NJ, Jewkes RK, Dunkle KL, McCarty F, Jama Shai N and Nduna M. Risk factors for unplanned and unwanted teenage pregnancies occurring over two years of follow-up among a cohort of young South African women. Glob Health Action. 2014;7:23719.

48. Hamdela B, G/mariam A and Tilahun T. Unwanted Pregnancy and Associated Factors among Pregnant Married Women in Hosanna Town, Southern Ethiopia. PLOS ONE. 2012 Jun 22;7(6):e39074. .

49. Dhakal S, Song JS, Shin DE, Lee TH, So AY and Nam EW. Unintended pregnancy and its correlates among currently pregnant women in the Kwango District, Democratic Republic of the Congo. Reproductive Health. 2016; 13:74.

50. Feyisso M, Girma A, Yimam H, Hailu S. Magnitude of Unintended Pregnancy and its Determinants among Pregnant Women Visiting Dilla University Referral Hospital. Journal of Women 's Health Care. 2017; 6(4):7.

51. Izugbara C. Socio-demographic risk factors for unintended pregnancy among unmarried adolescent Nigerian girls. South African Family Practice. 2015; 4;57(2):121-5.

52. Calvert C, Baisley K, Doyle AM, Maganja K, Changalucha J and Watson-Jones D. Risk factors for unplanned pregnancy among young women in Tanzania. Journal Family Planning Reproductive Health Care. 2013;39(4):e2.

53. Beguy D, Mumah J and Gottschalk L. Unintended Pregnancies among Young Women Living in Urban Slums: Evidence from a Prospective Study in Nairobi City, Kenya. PLoS ONE. 2014; 9(7): e101034.

54. Yenealem F, Niberet G. Prevalence and associated factors of unintended pregnancy among pregnant woman in Gondar town , North west Ethiopia. BMC Research Notes. 2019; 12:161.

55. Kassahun EA, Zeleke LB, Dessie AA, Gersa BG, Derseh HA, Arage MW, et al. Factors associated with unintended pregnancy among women attending antenatal care in Maichew town, northern Ethiopia, 2017. BMC Research Notes. 2019; 12:381. 
56. Fite RO, Mohammedamin A, Abebe TW. Unintended pregnancy and associated factors among pregnant women in Arsi Negele Woreda, West Arsi Zone, Ethiopia. BMC Research Notes. 2018;11(1):671.

57. Africa S, Haffejee F, Connor LO, Govender N, Reddy P, Sibiya MN, et al. Factors associated with unintended pregnancy among women attending a public health facility in KwaZulu-Natal, South Africa. South African Family Practice. 2017; $1(1): 1-5$.

58. Endriyas M, Eshete A, Mekonnen E, Misganaw T, Shiferaw M, Ayele S. Contraceptive utilization and associated factors among women of repro- ductive age group in southern Nations Nationalities and Peoples' Region, Ethiopia: crosssectional survey, mixed-methods. Contraceptive Reproductive Medicine. 2017;2:10.

59. Ayalew Y, Id G, Yitayew AE. Prevalence and determinant factors of unintended pregnancy among pregnant women attending antenatal clinics of Addis Zemen hospital; PLoS ONE. 2019.14(1): e0210206.

60. Aztlan EA, Foster DG, Upadhyay U. Subsequent unintended pregnancy among us women who receive or are denied a wanted abortion. J Mid-wifery Women's Health. 2018;63(1):45-52.

61. Megersa BS., Ojengbede oA.,Deckert A., Fawole OI. Factors associated with induced abortion among women of reproductive age attending selected health facilities in Addis Ababa, Ethiopia: a case control study.BMC Women's Health. 2020; 20:188.

62. Tilahun F, Dadi AF, Shiferaw G. Determinants of abortion among clients coming for abortion service at felegehiwot referral hospital, Northwest Ethiopia: a case control study. Contraceptive Reproductive Medicine. 2017;2(1):11. .

63. Lamina MA. Prevalence of abortion and contraceptive practice among women seeking repeat induced abortion in Western Nigeria. BMC Women's Health. 2020;20:188.

64. Sibanda A, Woubalem Z, Hogan DP, Lindstrom DP. The proximate determinants of the decline to below replacement fertility in Addis Ababa, Ethiopia. Studies of Family Planning. 2003;34(1):1-7.

65. Gurmu E, Mace R. Fertility decline driven by poverty: the case of Addis Ababa, Ethiopia. Journal of Biosocial Science. 2008;40(3):339-58.

66. Gilano G, Hailegebreal S. Determinants of abortion among youth 15-24 in Ethiopia: A multilevel analysis based on EDHS 2016. PLoS ONE. 2021;16(3):e0248228.

67. Abera GB, Berhanu B, Kahsay AB, Gebru HB, Aregay A. Assessment of determinants of induced abortion among child bearing age women attending maternal and child health clinic in Mekelle town, Tigray, Ethiopia: A cross sectional study. International Journal of Pharmaceutical Science Research. 2012;3: 4745-4756.

68. Mohammed F, Musa A and Amano A. Prevalence and determinants of unintended pregnancy among pregnant woman attending ANC at Gelemso General Hospital, Oromiya Region, East Ethiopia: a facility based cross-sectional study. BMC Womens Health. 2016; 4:16.

69. Mekie $\mathrm{M}$ et al. The level and deriving factors of repeated induced abotion in Ethiopia: a systematc review and meta anayisis. Heliyon. 2021:7 e05984.

\section{Figures}




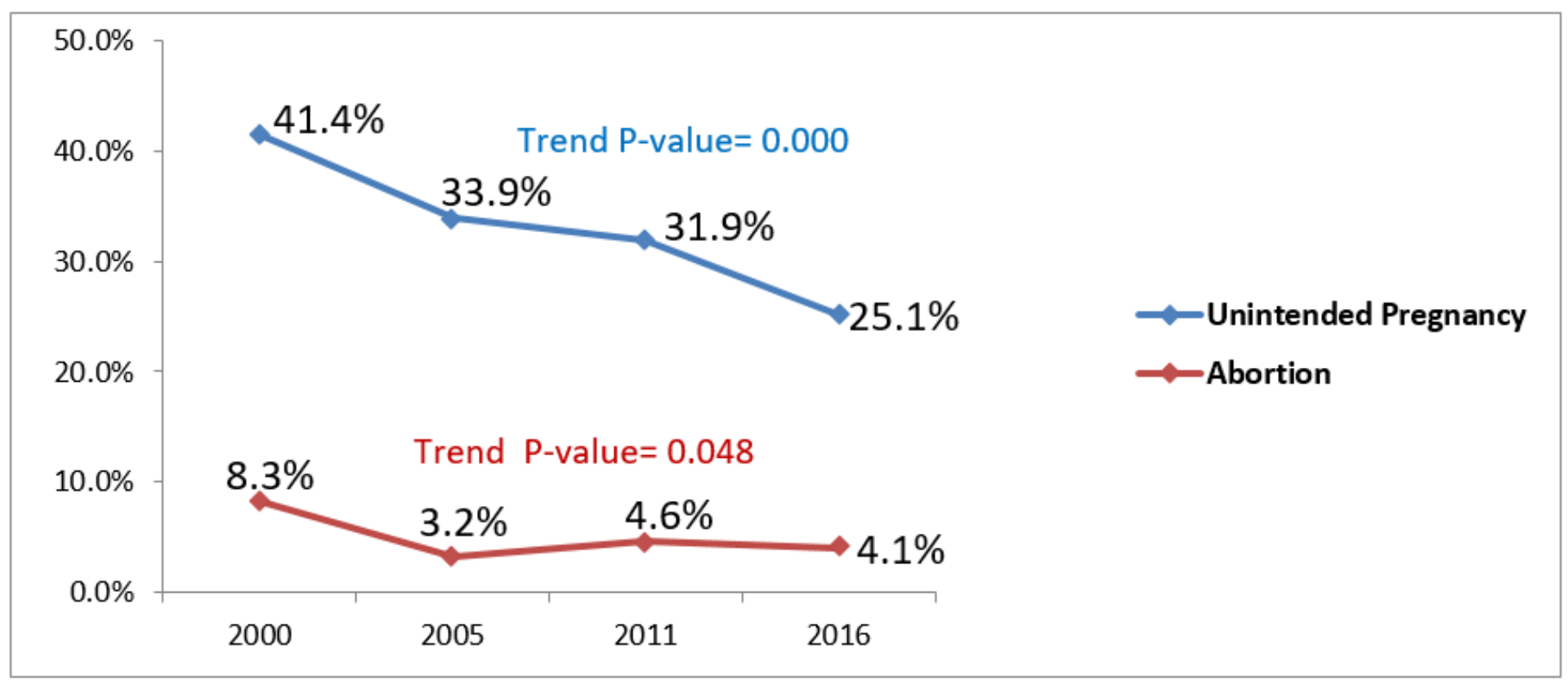

Figure 1

Trend of unintended pregnancy and abortion among adolescents in Ethiopia, 2000-2016. 